\title{
Charged Lepton Flavor Violation search on Mu2e at Fermilab
}

在费米实验室Mu2e 实验上寻找带电轻子味破坏

\author{
Zhengyun You（尤郑昀） \\ Sun Yat-Sen University（中山大学） \\ Workshop on Intensity Frontier of Particle Physics \\ 9/9/2018, Institute of Theoretical Physics, CAS, Beijing
}




\section{Overview}

- Charged Lepton Flavor Violation (CLFV)

- CLFV measurements

- Muon to electron conversion search on Mu2e

- The experimental apparatus

- Current status

- Next plan - Muzell 


\section{Flavor Violation}

Standard Model of Elementary Particles

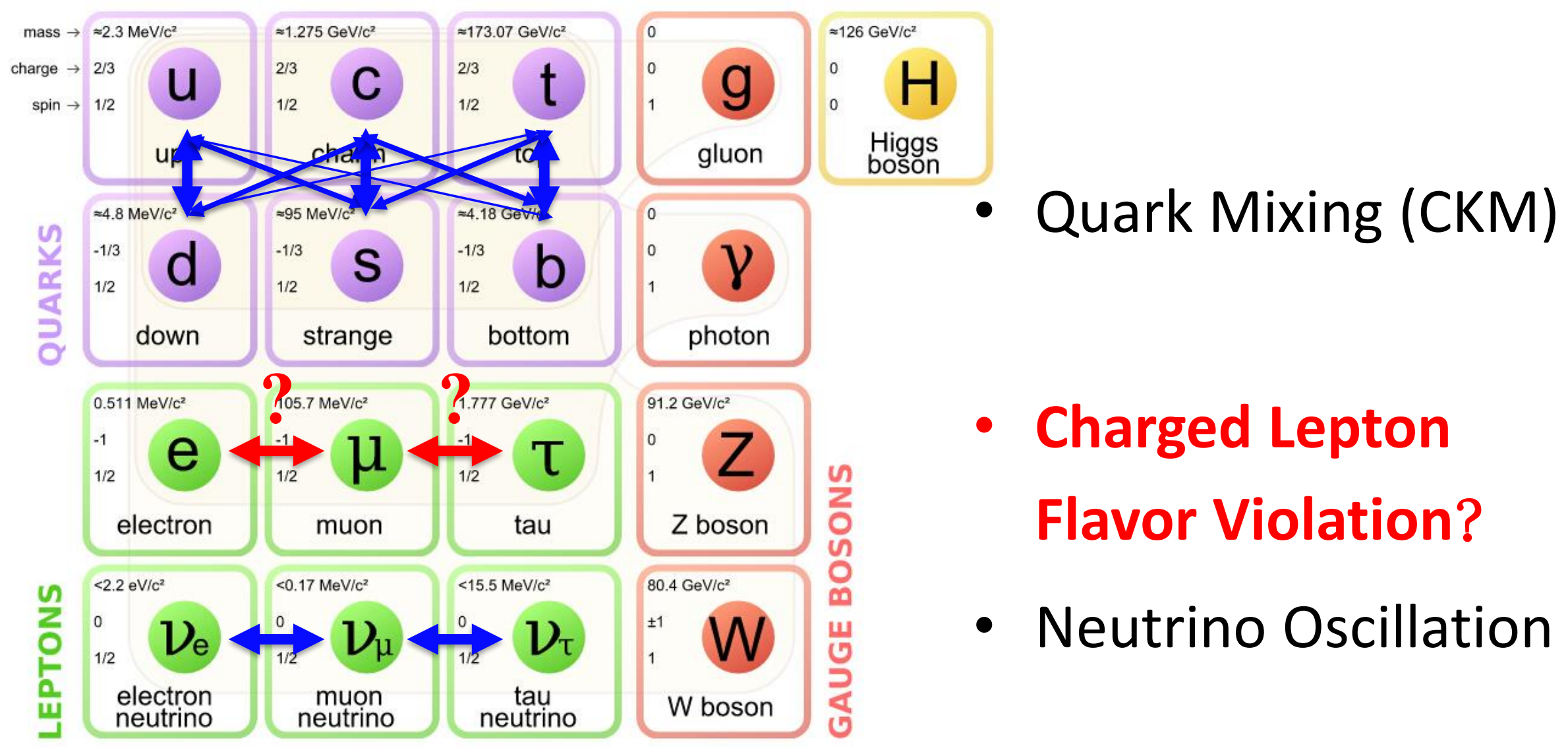




\section{CLFV and BSM}

- Ordinary muon decay

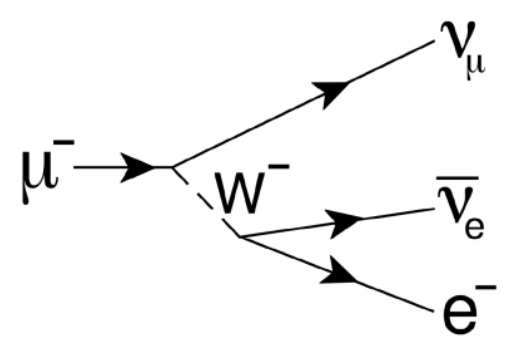

Lepton Flavor Conservation

\begin{tabular}{|c|c|c|c|c|c|}
\hline & muon & & $\begin{array}{l}\text { nuon } \\
\text { eutrino }\end{array}$ & electron & antineutrino \\
\hline equation: & $\mu$ & $\rightarrow$ & $v_{\mu}$ & $+e^{-}$ & $+\bar{v}_{e}$ \\
\hline $\begin{array}{l}\text { electron } \\
\text { number: }\end{array}$ & 0 & $=$ & 0 & + & -1 \\
\hline $\begin{array}{c}\text { muon } \\
\text { number: }\end{array}$ & 1 & $=$ & 1 & + & + \\
\hline
\end{tabular}

- CLFV could exist but is too tiny to be measurable

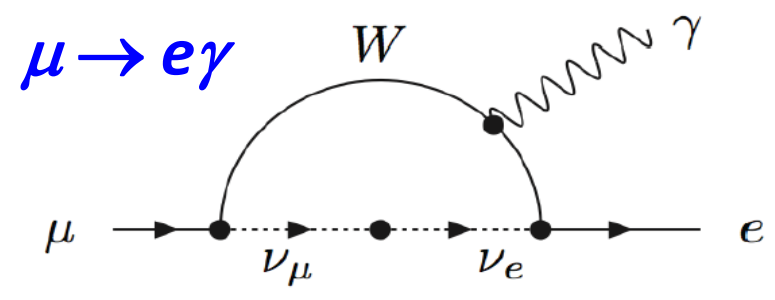

$$
\begin{gathered}
\mathcal{B}(\mu \rightarrow e \gamma)=\frac{3 \alpha}{32 \pi}\left|\sum_{i=2,3} U_{\mu i}^{*} U_{e i} \frac{\Delta m_{1 i}^{2}}{M_{W}^{2}}\right|^{2}<10-54 \\
\text { PMNS unitary, } \sum \mathrm{U}_{\mu i}{ }^{*} \mathrm{U}_{\mathrm{ei}}=0
\end{gathered}
$$

- Any CLFV observation implies New Physics Beyond the Standard Model (BSM) 


\section{CLFV searches}

\begin{tabular}{|c|c|c|c|}
\hline \multirow[b]{2}{*}{$\mu^{-}+\mathbf{N} \rightarrow \mathbf{e}^{-}+\mathbf{N}$} & Process & Current Limit & Next Generation exp \\
\hline & $\begin{array}{l}\tau \rightarrow \mu \eta \\
\tau \rightarrow \mu \gamma \\
\tau \rightarrow \mu \mu \mu \\
\tau \rightarrow \text { eee }\end{array}$ & $\begin{array}{l}\mathrm{BR}<6.5 \mathrm{E}-8 \\
\mathrm{BR}<6.8 \mathrm{E}-8 \\
\mathrm{BR}<3.2 \mathrm{E}-8 \\
\mathrm{BR}<3.6 \mathrm{E}-8\end{array}$ & $10^{-9}-10^{-10}($ Belle II) \\
\hline \multirow{2}{*}{$\begin{array}{l}\text { One of the most } \\
\text { effective channels }\end{array}$} & $\begin{array}{l}\mathrm{K}_{\mathrm{L}} \rightarrow \mathrm{e} \mu \\
\mathrm{K}^{+} \rightarrow \pi^{+} \mathrm{e}^{-} \mu^{+}\end{array}$ & $\begin{array}{l}\mathrm{BR}<4.7 \mathrm{E}-12 \\
\mathrm{BR}<1.3 \mathrm{E}-11\end{array}$ & \\
\hline & $\begin{array}{l}\mathrm{B}^{0} \rightarrow \mathrm{e} \mu \\
\mathrm{B}^{+} \rightarrow \mathrm{K}^{+} \mathrm{e} \mu\end{array}$ & $\begin{array}{l}\mathrm{BR}<7.8 \mathrm{E}-8 \\
\mathrm{BR}<9.1 \mathrm{E}-8\end{array}$ & \\
\hline$\mu$ channels & $\begin{array}{l}\mu^{+} \rightarrow \mathrm{e}^{+} \gamma \\
\mu^{+} \rightarrow \mathrm{e}^{+} \mathrm{e}^{+} \mathrm{e}^{-} \\
\mu \mathrm{N} \rightarrow \mathrm{eN}\end{array}$ & $\begin{array}{l}\mathrm{BR}<4.2 \mathrm{E}-13 \\
\mathrm{BR}<1.0 \mathrm{E}-12 \\
\mathrm{R}_{\mu \mathrm{e}}<7.0 \mathrm{E}-13\end{array}$ & $\begin{array}{c}10^{-14} \text { (MEG) } \\
10^{-16} \text { (PSI) } \\
10^{-17} \text { (Mu2e, COMET) }\end{array}$ \\
\hline
\end{tabular}




\section{Probing New Physics with CLFV}
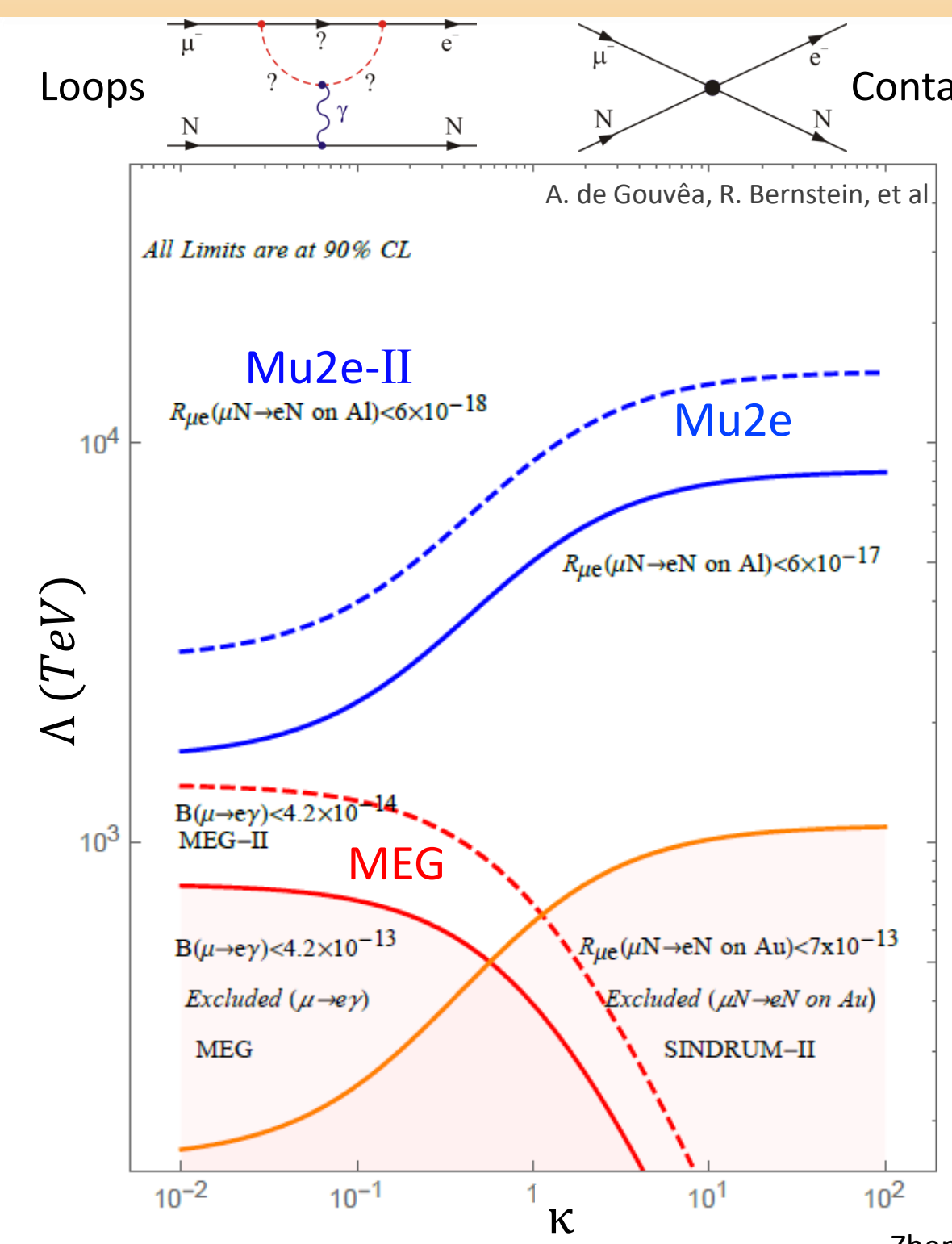

Zhengyun You (SYSU)
Effective CLFV Lagrangian

$L=\frac{m_{\mu}}{(\kappa+1) \Lambda^{2}} \bar{\mu} R \sigma_{\mu v} e_{L} F_{\mu v}+\frac{\kappa}{(\kappa+1) \Lambda^{2}} \bar{\mu}_{L} \gamma_{\mu} e_{L} \sum_{q=u, d} \bar{q}_{L} \gamma^{\mu} q_{L}$

- Relative strength $\kappa$, mass scale $\Lambda$

- 'Loops', electromagnetic operator, $\kappa<<1$, can be probed by $\mu \rightarrow e \gamma$ and $\mu N \rightarrow e N$

- 'Contact terms', direct coupling between quarks and leptons, $\kappa \gg>1$, accessible by $\mu N \rightarrow e N$

- Mu2e will probe mass scale $\Lambda$ 2,000 10,000 TeV

Beyond the reach of accelerators 


\section{CLFV and Theoretic Models}

$\mu^{-}+\mathrm{N} \rightarrow \mathrm{e}^{-}+\mathrm{N}$

One of the most effective channels

사순 Large effects

수 Visible, but small No sizable effect

\begin{tabular}{|l|c|c|c|c|c|c|c|}
\hline & AC & RVV2 & AKM & $\delta$ LL & FBMSSM & LHT & RS \\
\hline \hline$D^{0}-\bar{D}^{0}$ & $\star \star \star$ & $\star$ & $\star$ & $\star$ & $\star$ & $\star \star \star$ & $?$ \\
\hline$\epsilon_{K}$ & $\star$ & $\star \star \star$ & $\star \star \star$ & $\star$ & $\star$ & $\star \star$ & $\star \star \star$ \\
\hline$S_{\psi \phi}$ & $\star \star \star$ & $\star \star \star$ & $\star \star \star$ & $\star$ & $\star$ & $\star \star \star$ & $\star \star \star$ \\
\hline \hline$S_{\phi K}$ & $\star \star \star$ & $\star \star$ & $\star$ & $\star \star \star$ & $\star \star \star$ & $\star$ & $?$ \\
\hline$A_{\mathrm{CP}}\left(B \rightarrow X_{s} \gamma\right)$ & $\star$ & $\star$ & $\star$ & $\star \star \star$ & $\star \star \star$ & $\star$ & $?$ \\
\hline$A_{7,8}\left(B \rightarrow K^{*} \mu^{+} \mu^{-}\right)$ & $\star \star$ & $\star$ & $\star$ & $\star \star \star$ & $\star \star \star$ & $\star \star$ & $?$ \\
\hline$A_{9}\left(B \rightarrow K^{*} \mu^{+} \mu^{-}\right)$ & $\star \star$ & $\star$ & $\star$ & $\star$ & $\star$ & $\star$ & $?$ \\
\hline$B \rightarrow K^{(\star)} \nu \bar{\nu}$ & $\star \star$ & $\star$ & $\star$ & $\star$ & $\star$ & $\star$ & $\star$ \\
\hline$B_{s} \rightarrow \mu^{+} \mu^{-}$ & $\star \star \star$ & $\star \star \star$ & $\star \star \star$ & $\star \star \star$ & $\star \star \star$ & $\star$ & $\star$ \\
\hline$K^{+} \rightarrow \pi^{+} \nu \bar{\nu}$ & $\star \star$ & $\star$ & $\star$ & $\star$ & $\star$ & $\star \star \star$ & $\star \star \star$ \\
\hline$K_{L} \rightarrow \pi^{0} \nu \bar{\nu}$ & $\star \star$ & $\star$ & $\star$ & $\star$ & $\star$ & $\star \star \star$ & $\star \star \star$ \\
\hline$\mu \rightarrow e \gamma$ & $\star \star \star$ & $\star \star \star$ & $\star \star \star$ & $\star \star \star$ & $\star \star \star$ & $\star \star \star$ & $\star \star \star$ \\
\hline$\tau \rightarrow \mu \gamma$ & $\star \star \star$ & $\star \star \star$ & $\star$ & $\star \star \star$ & $\star \star \star$ & $\star \star \star$ & $\star \star \star$ \\
\hline$\mu+N \rightarrow e+N$ & $\star \star \star$ & $\star \star \star$ & $\star \star \star$ & $\star \star \star$ & $\star \star \star$ & $\star \star \star$ & $\star \star \star$ \\
\hline$d_{n}$ & $\star \star \star$ & $\star \star \star$ & $\star \star \star$ & $\star \star$ & $\star \star \star$ & $\star$ & $\star \star \star$ \\
\hline$d_{e}$ & $\star \star$ & $\star \star \star$ & $\star \star$ & $\star$ & $\star \star \star$ & $\star$ & $\star \star \star$ \\
\hline$(g-2)_{\mu}$ & $\star \star$ & $\star \star \star$ & $\star \star$ & $\star \star \star$ & $\star \star \star$ & $\star$ & $?$ \\
\hline
\end{tabular}

Table 8: "DNA" of flavour physics effects for the most interesting observables in a selection of SUSY and non-SUSY models $\star \star \star$ signals large effects, $\star \star$ visible but small effects and $\star$ implies that the given model does not predict sizable effects in that observable.

W. Altmannshofer, A.J.Buras, S.Gori, P.Paradisi, D.M.Straub, NPB 830, 17 (2010) 


\section{Current Best Limit in $\mu N \rightarrow e N$}

W. Bertl et al, Eur. Phys. J. C 47, 337-346 (2006)

- Current best limit

- by SINDRUM II at PSI

$-\mathrm{R}_{\mu e}(\mathrm{Au})<7 \times 10^{-13}$ (90\%C.L.)

- Au target: different electron energy endpoint than Al
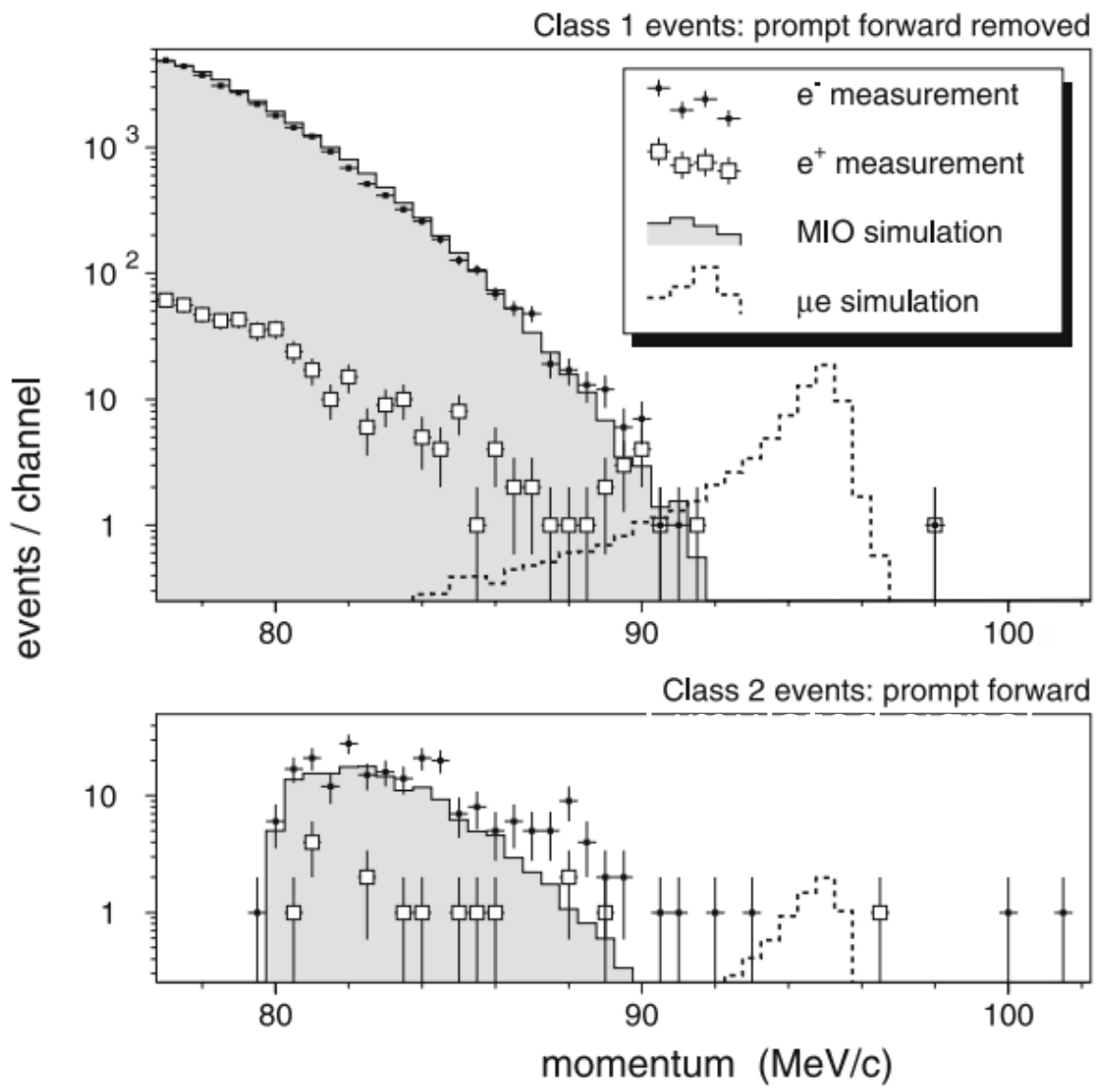

Fig. 11. Momentum distributions of electrons and positrons for the two event classes. Measured distributions are compared with the results of simulations of muon decay in orbit and $\mu-e$ conversion 


\section{$\mu \rightarrow \mathbf{e} \gamma \& \mu N \rightarrow e N$}

\section{$\mu \rightarrow$ ev}

- Signal particles $\leq 53 \mathrm{MeV}$

- high background region

- Signal: combination of particles

- Background rejection: cuts on track combinations

- Accidental coincidences limit usable muon rate

- Continuous beam

- $\mu+$

- Capture rates: $\Gamma_{\mu+}=0$

\section{$\mu \mathrm{N} \rightarrow \mathrm{eN}$}

- Signal particle at $105 \mathrm{MeV}$

- Away from Michel peak

- Signal: single track

- Fewer handles to reject background

- Coincidences not a problem

- Use pulsed beam

- $\quad$ suppress background between pulses

- Need cosmic ray veto

- $\mu-$

- Capture rates: $\Gamma_{\mu-} \sim Z^{4}$

- Potential background 
- Located at Fermi National Laboratory

- One of the U.S. Intensity Frontier top priority projects

- Cost “\$27j ivi dólilar"s (JE accountingi)

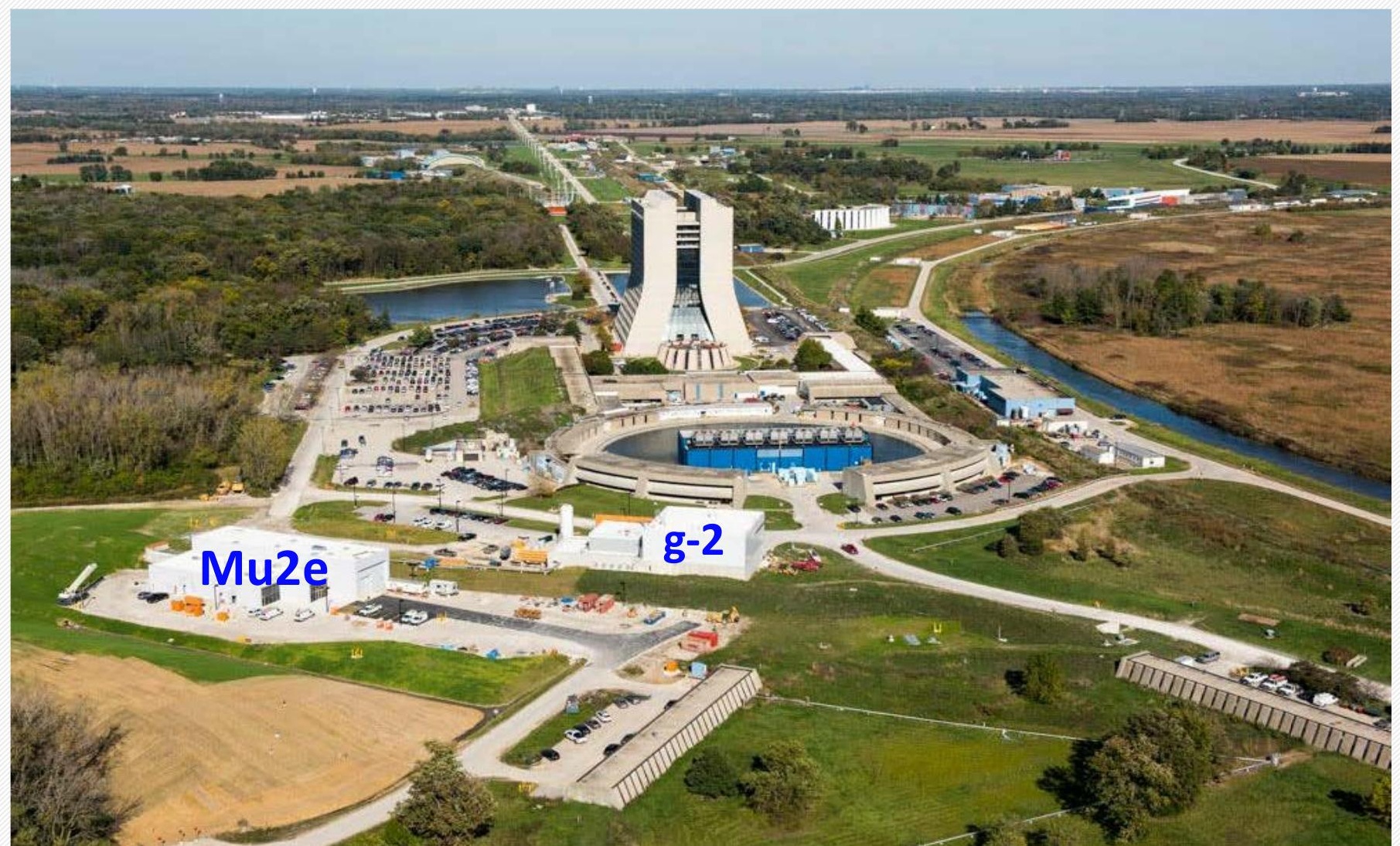




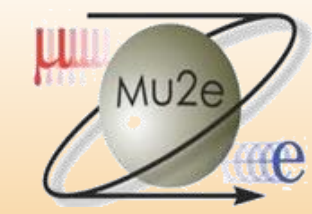

\section{Mu2e Building}

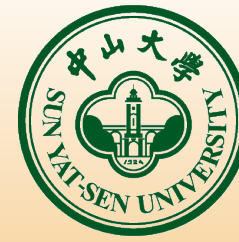

- Civil construction completed (2017/3)
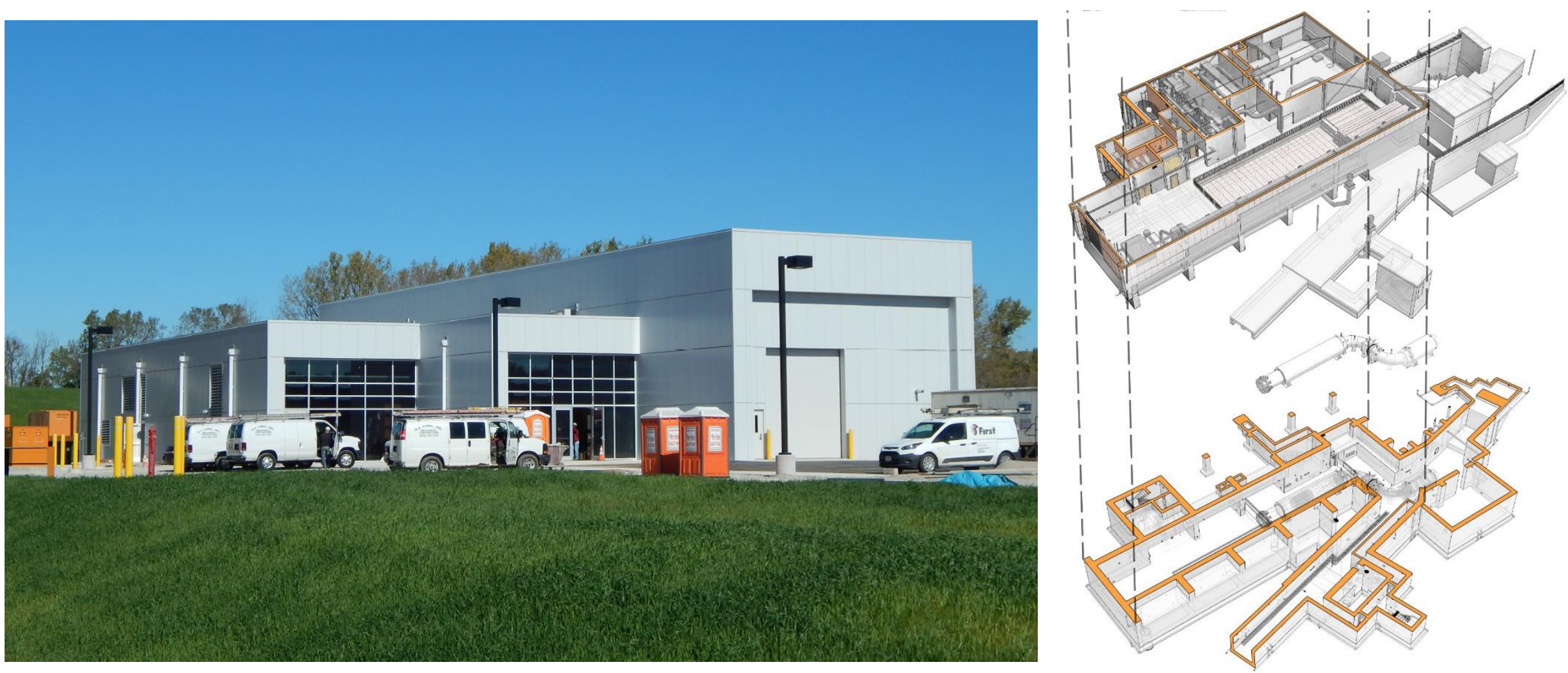


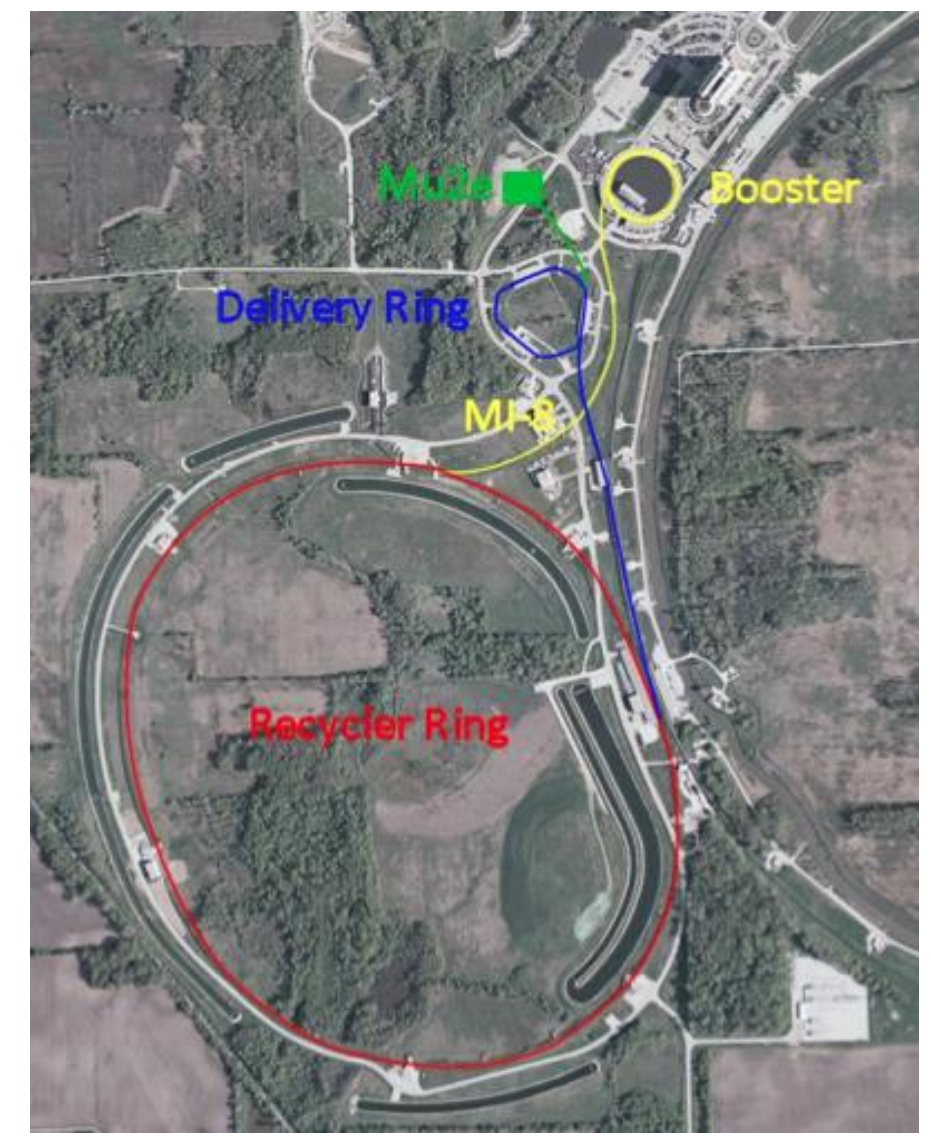

- Use existing accelerator infrastructure

- Booster provides $8 \mathrm{GeV}$ protons

- Recycler stacks protons into 4 bunches

- Deliver Ring takes one bunch from Recycler

- Run simultaneously with neutrino programs NOvA. 


\section{Beam Time Structure}

1695 ns peak-to-peak

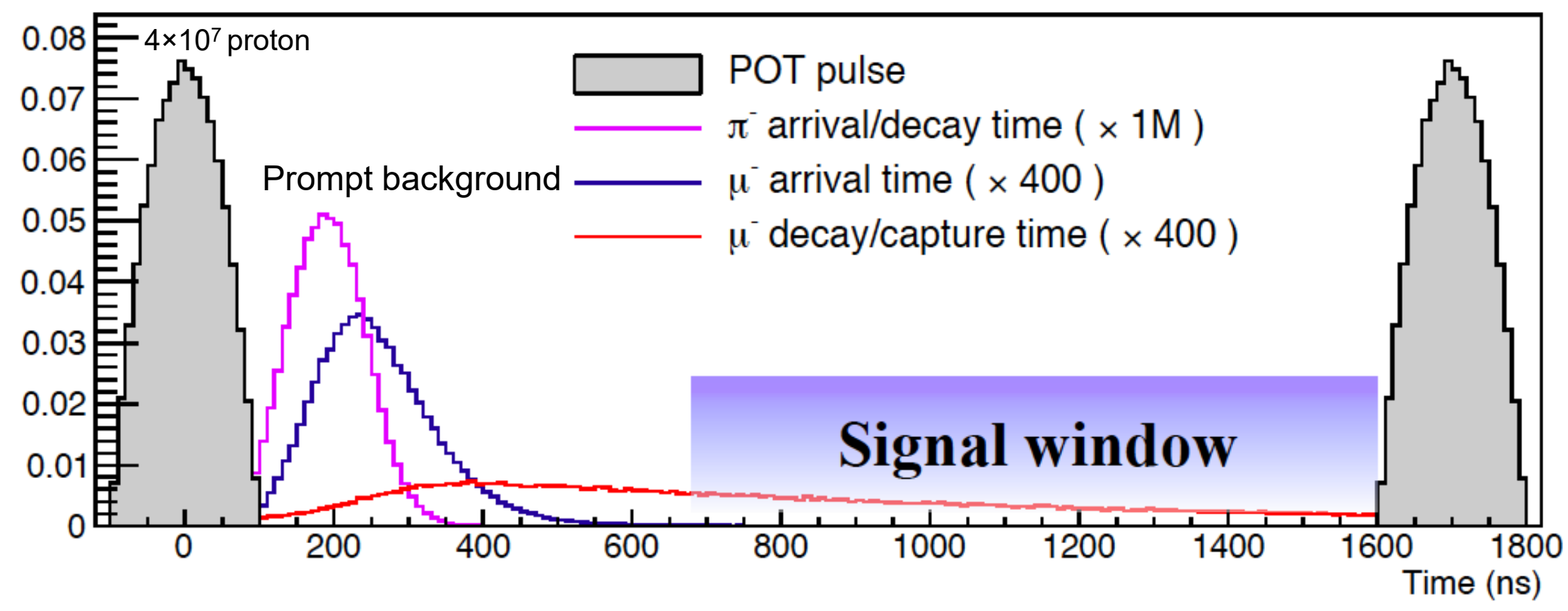

- $4 \times 10^{7}$ protons per bunch, bunch spacing 1695 ns

- 700 ns delay before 1 ms live gate

- Extinction factor (Out-Of-Time proton rate) $<10^{-10}$. 
- $>220$ scientists from 38 institutions

- Sun Yat-Sen University joined Mu2e in 2016

Argonne National Laboratory,

Boston University,

University of California Berkeley, University of California Irvine, California Institute of Technology, City University of New York, Joint Institute of Nuclear Research Dubna, Duke University, Fermi National Accelerator Laboratory, Laboratori Nazionale di Frascati, University of Houston, Helmholtz-Zentrum Dresden-Rossendorf, INFN Genova, Institute for High Energy Physics, Protvino, Kansas State University, Lawrence Berkeley National Laboratory, INFN Lecce, University Marconi Rome, Lewis University, University of Liverpool,
University College London, University of Louisville, University of Manchester, University of Michigan, University of Minnesota, Muon Inc.,

Northwestern University, Institute for Nuclear Research Moscow, INFN Pisa,

Northern Illinois University, Purdue University,

Rice University, Sun Yat-Sen University, University of South Alabama, Novosibirsk State University Budker Institute of Nuclear Physics, University of Virginia, University of Washington, Yale University
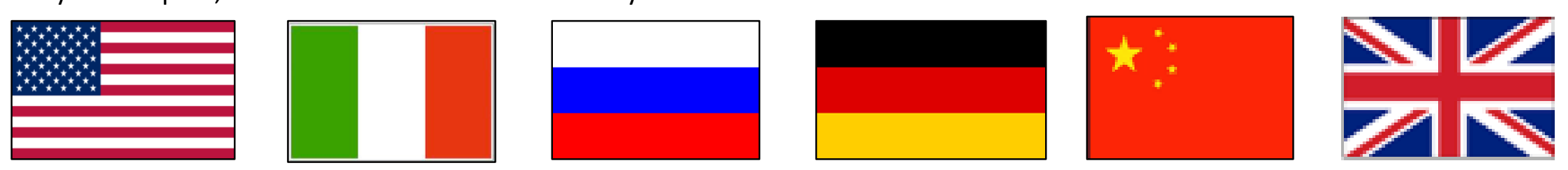


\section{$\mu \mathrm{N} \rightarrow \mathrm{eN}$ Conversion}

\section{What to measure}

- The ratio of muon to electron conversions to the number of muon captures by nuclei

$$
R_{\mu e}=\frac{\mu^{-}+_{13}^{27} A l \rightarrow e^{-}+_{13}^{27} A l}{\mu^{-}+_{13}^{27} A l \rightarrow \text { nuclear capture }}
$$

- Signal: Neutrinoless conversion of a muon to electron in the field of a nucleus

\section{Coherent Conversion}

- Experimental signature

- Mono-energetic electron

$-E_{e}=m_{\mu}-E_{\text {bind }}-E_{\text {recoil }}$

- For Al, $\mathrm{E}_{\mathrm{e}}=104.97 \mathrm{MeV}$

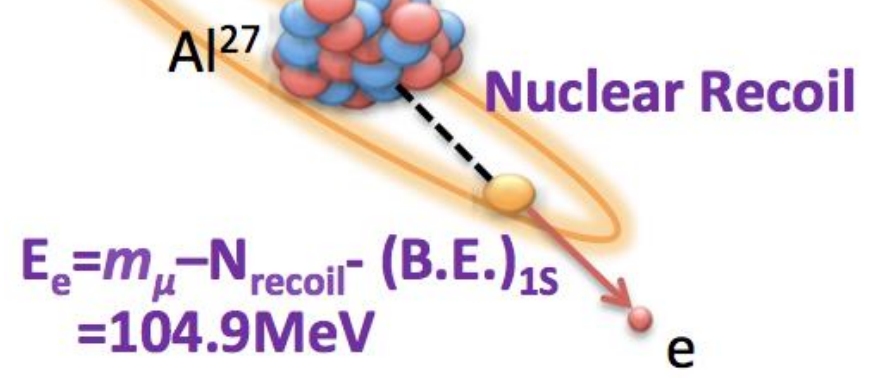




\section{Mu2e Apparatus}

Production

Solenoid

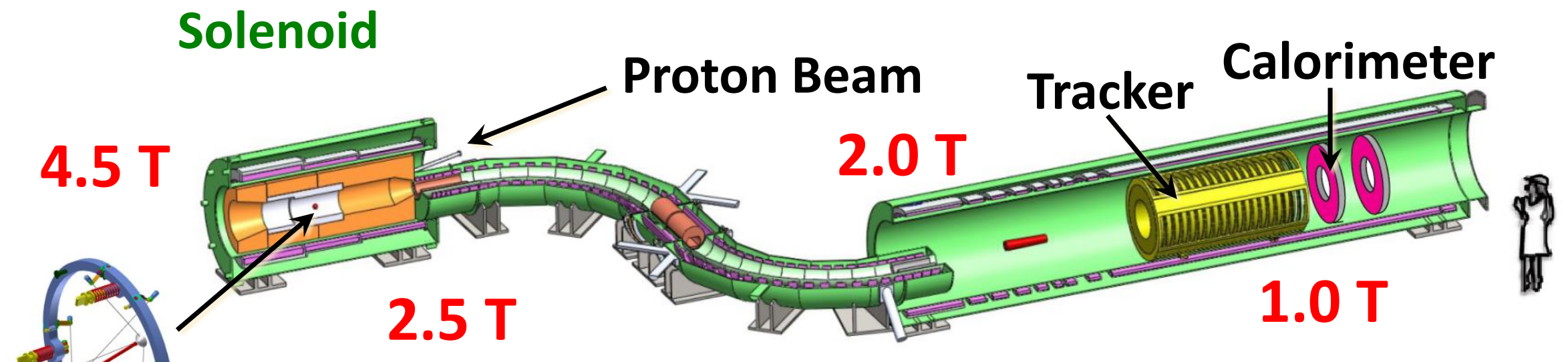

\section{Production} Target

Transport Solenoid

Detector Solenoid

Three functional solenoids with graded fields $(4.5 \mathrm{~T}-1 \mathrm{~T})$

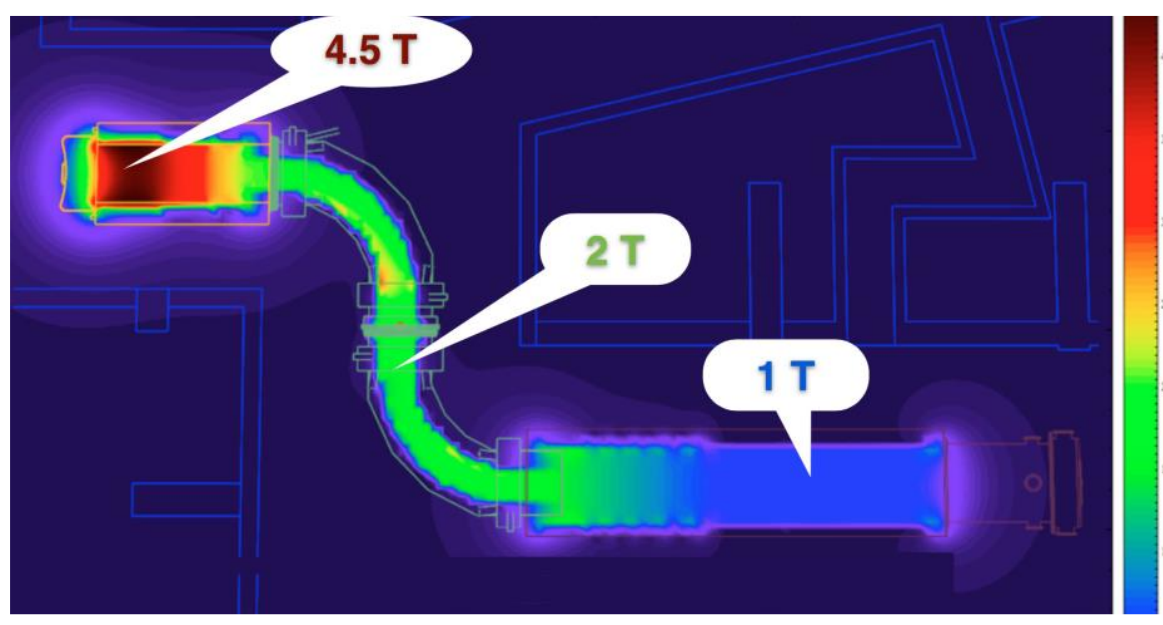




\section{Target and Shield}

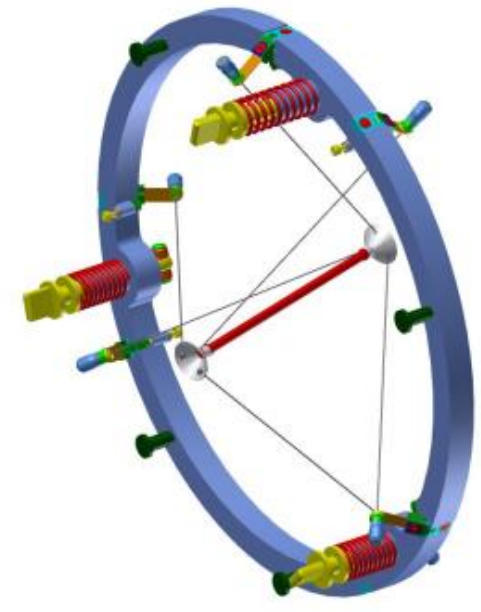

- Production Target

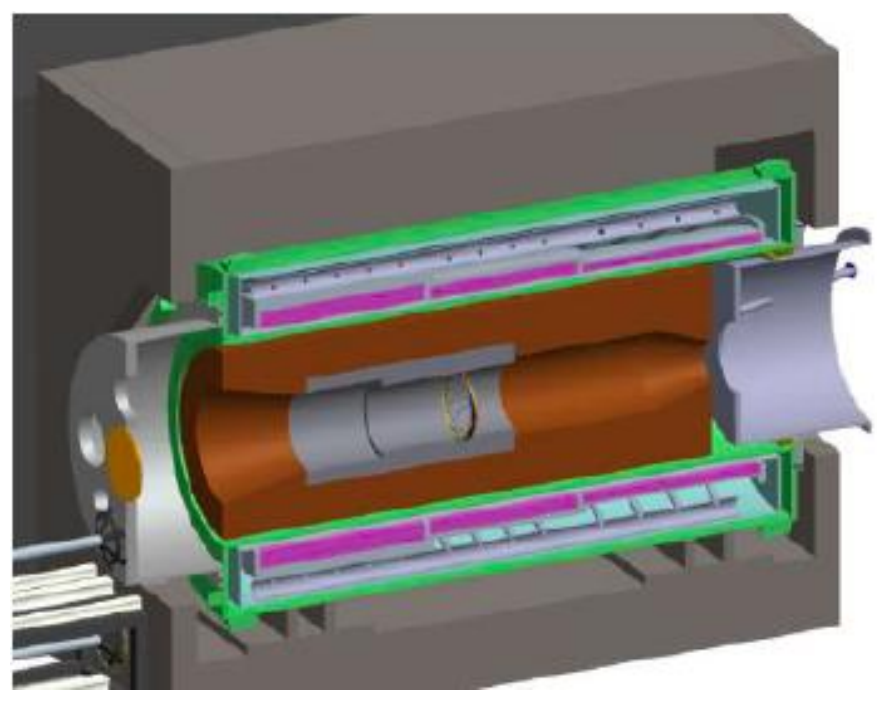

- Protons + target $\rightarrow \pi+\mathrm{X}$; $\pi$ decay into muons

- High A and high density material Tungsten to maximize muons production

- High melting temp, radiative cooling ( $1600 \circ \mathrm{C})$, with $8 \mathrm{~kW}$ beam (700w in target)

- Heat Radiation Shield

- To protect superconductor of PS and upstream TS

- To limit heat load and radiation damage

- $\sim 25$ tons of Bronze 


\section{Transport Solenoid}

- Gradient magnetic field from 2.5 T to 2.0 T

- S-shaped magnetic channel to transmit lowmomentum negatively charged particles in helical trajectories

- Collimators to remove positively charged and high-momentum particles
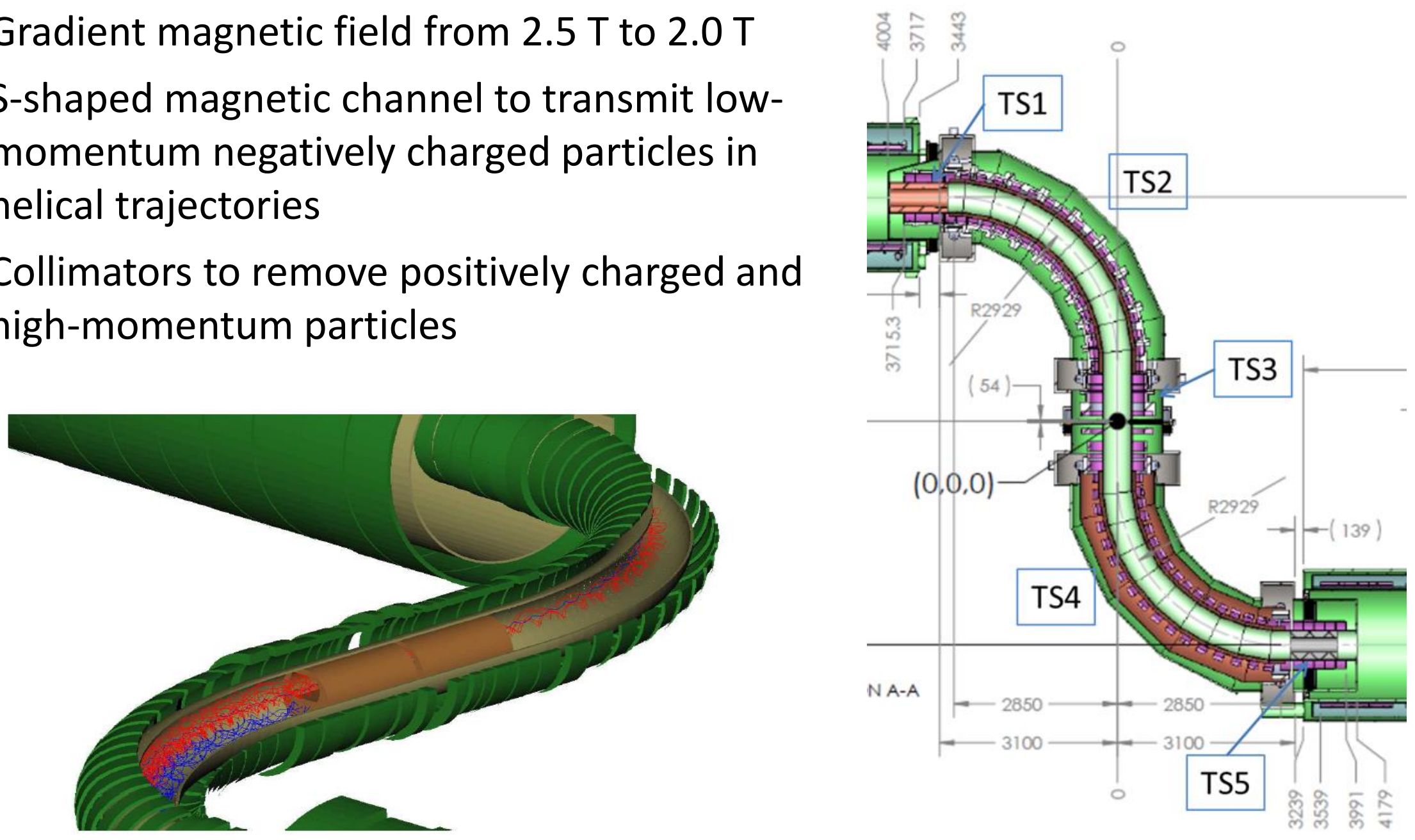


\section{Detector Solenoid}
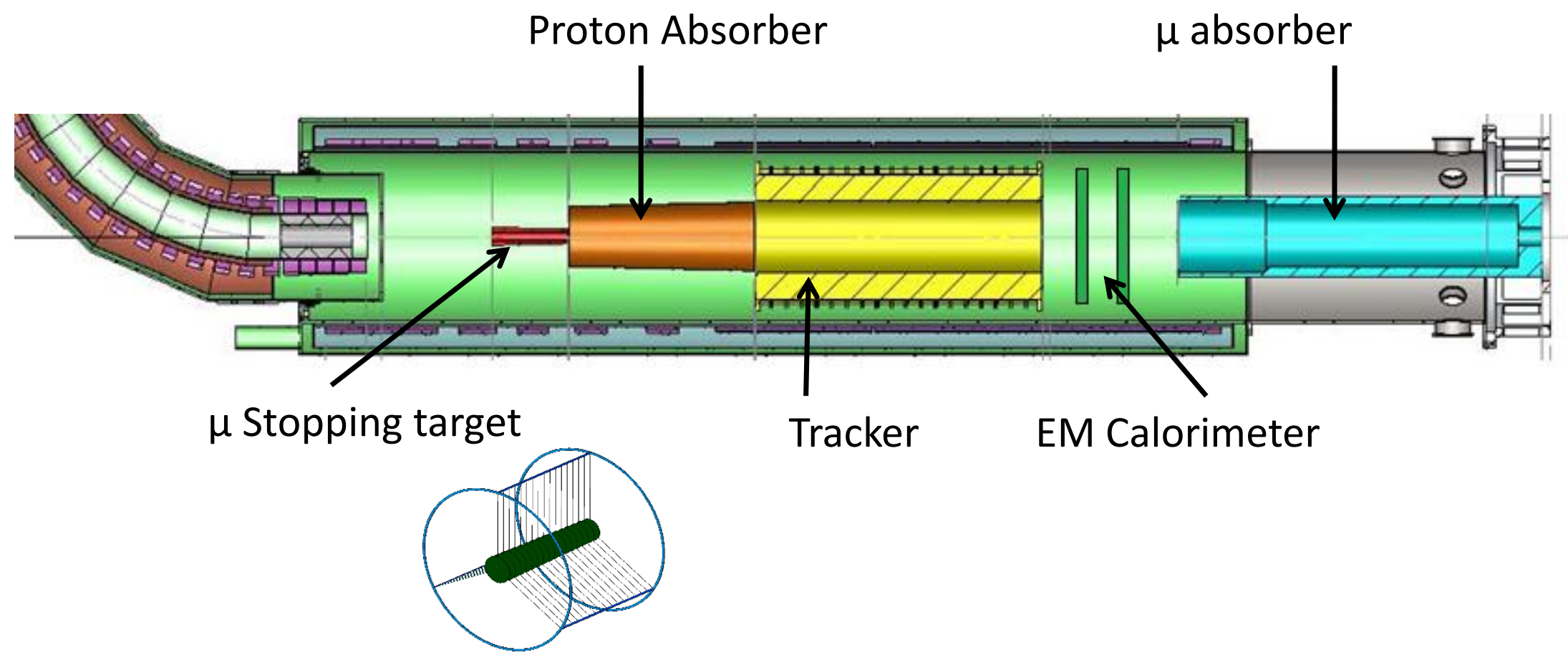

- Stopping target to capture muons

- Graded magnetic field from $2 \mathrm{~T}$ to $1 \mathrm{~T}$, captures conversion electrons with bigger acceptance

- Tracker and Calorimeter in a uniform field to reconstruct and identify electrons. 
Tracker Structure
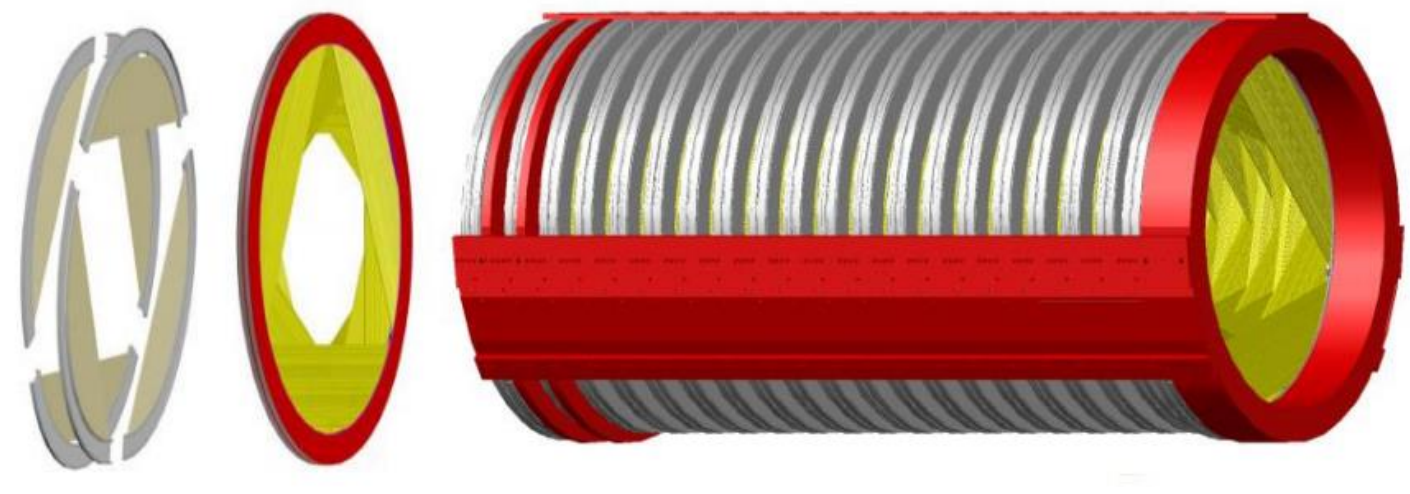

\section{$105 \mathrm{MeV}$}

Conversion electrons

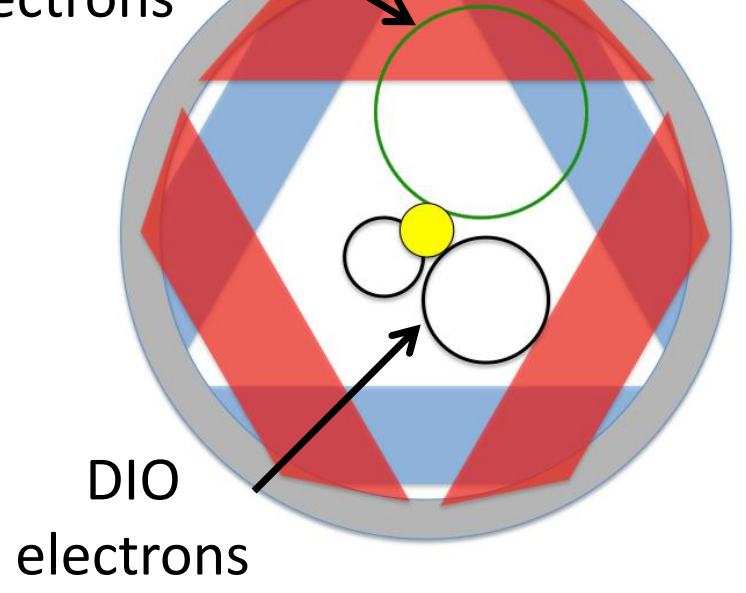

Straw Tube

- $5 \mathrm{~mm}$ diameter straw drift tubes

- $15 \mu \mathrm{m}$ Mylar walls, filled with $\mathrm{Ar}: \mathrm{CO}_{2}$ (80:20)

- 18 stations, 2 planes/station, 6 panels /plane

- Blind to beam flash and >97\% DIO

- Expect $100 \mu \mathrm{m}$ hit resolution

- Momentum resolution < 180 keV/c (@105MeV/c) 


\section{Calorimeter}
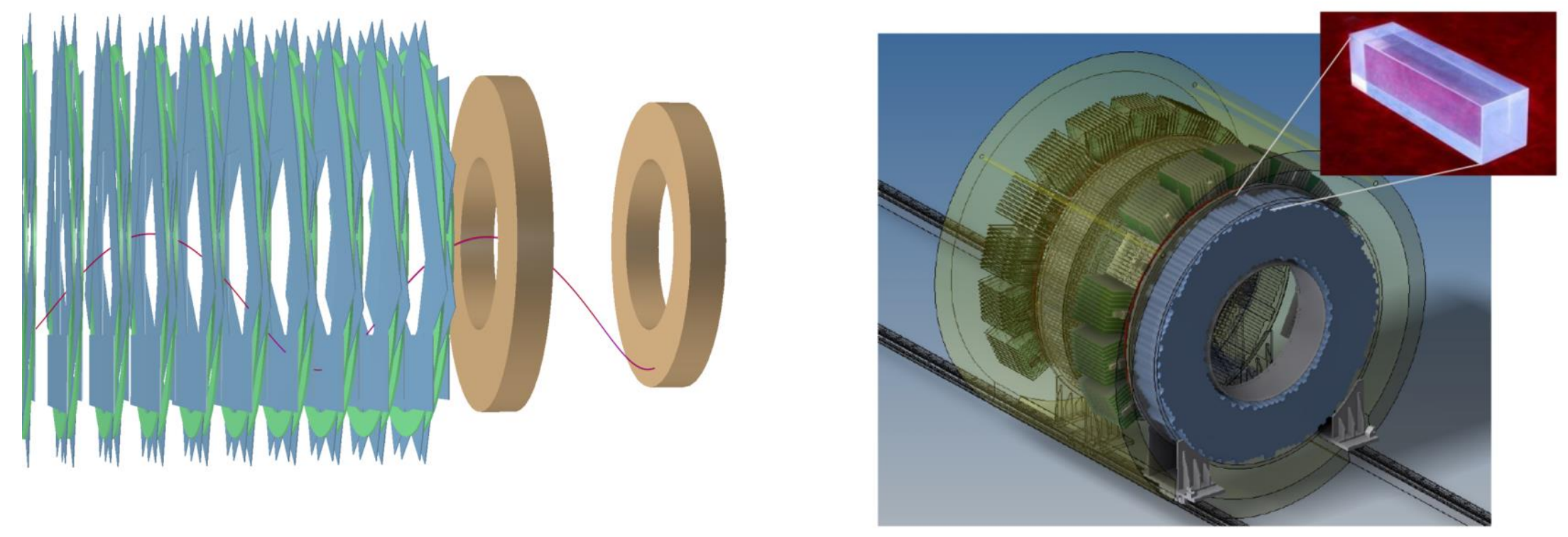

- Two disks separated by $70 \mathrm{~cm}(1 / 2 \lambda)$, 674 Csl crystals/disk

- Provides independent energy $\left(\sigma_{\mathrm{E}} / \mathrm{E}<5 \%\right)$, time $\left(\sigma_{\mathrm{t}}<0.5 \mathrm{~ns}\right)$ and position $\left(\sigma_{\mathrm{pos}} \sim 1 \mathrm{~cm}\right)$ measurements

- Particle ID, Cosmic Ray rejection, tracking seed

- Independent trigger 

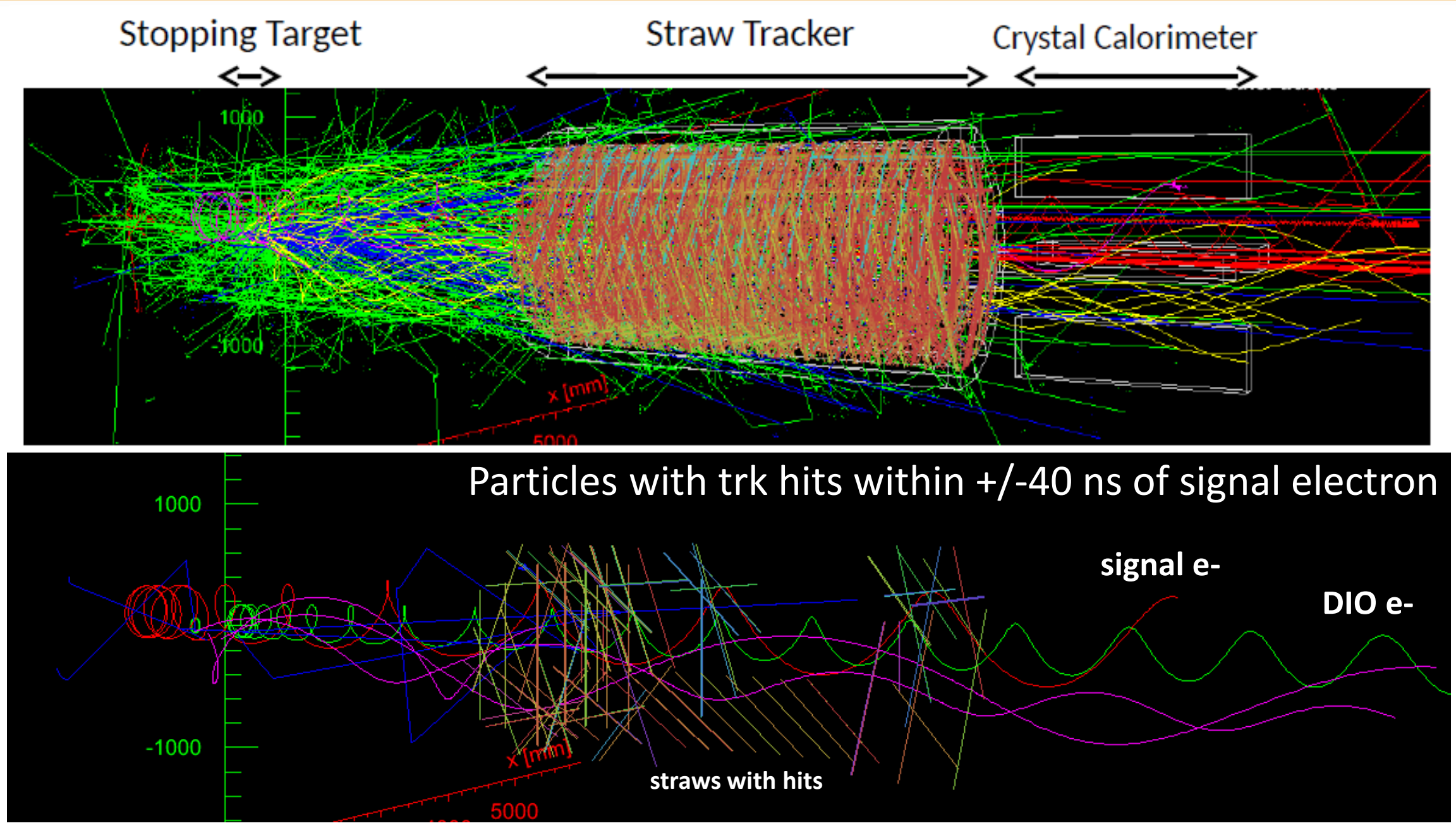

To find a signal electron near $105 \mathrm{MeV} / \mathrm{c}$ from a bunch of track candidates 


\section{Background(1): Intrinsic}

$>$ Muon Decay-in-orbit (DIO)

- $\mu \rightarrow \mathrm{e} v_{\mu} \overline{v_{e}}$

- Dominant background

- 39\% of stopped muons

- DIO Rate $\sim\left(\mathrm{E}_{\mathrm{MAX}}-\mathrm{E}_{\mathrm{e}}\right)^{5}, \mathrm{E}_{\mathrm{MAX}} \sim 105 \mathrm{MeV}$

- Requires good energy resolution

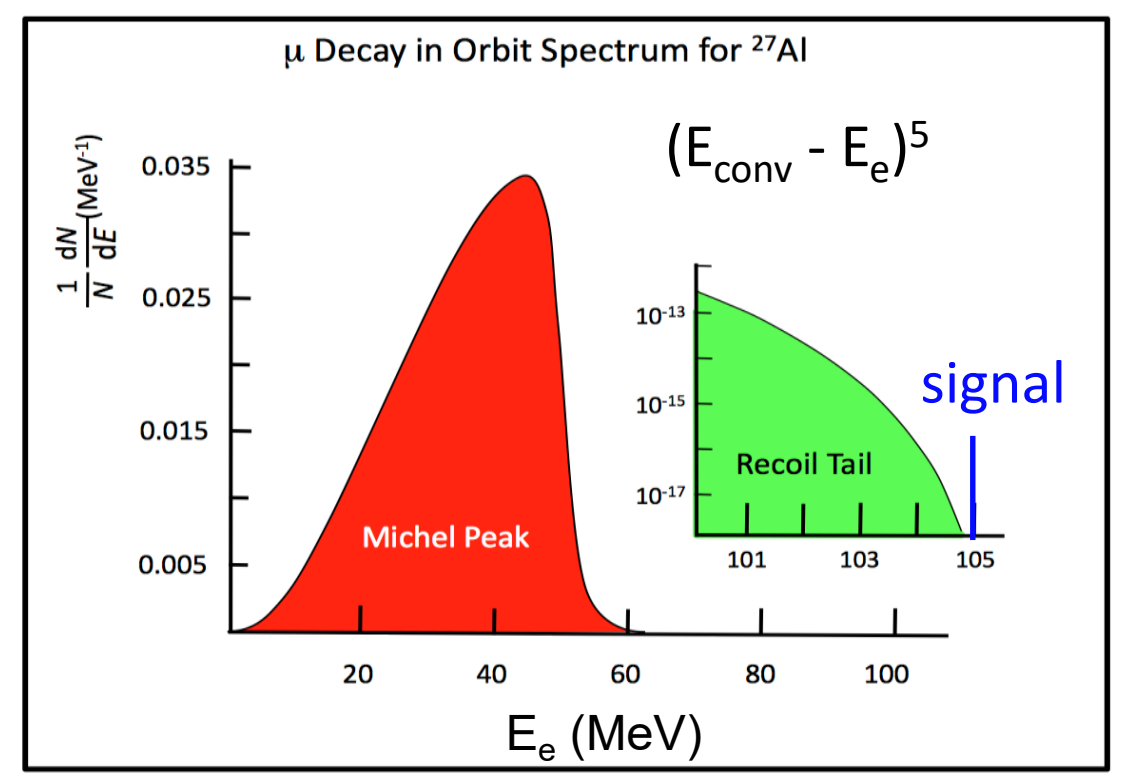

$>$ Radiative Muon Capture (RMC)

- $\mu \mathrm{N} \rightarrow \gamma v_{\mu} \mathrm{N}^{\prime *}$

- $61 \%$ of stopped muons

- $3 \mathrm{MeV}$ under DIO end point

- Negligible

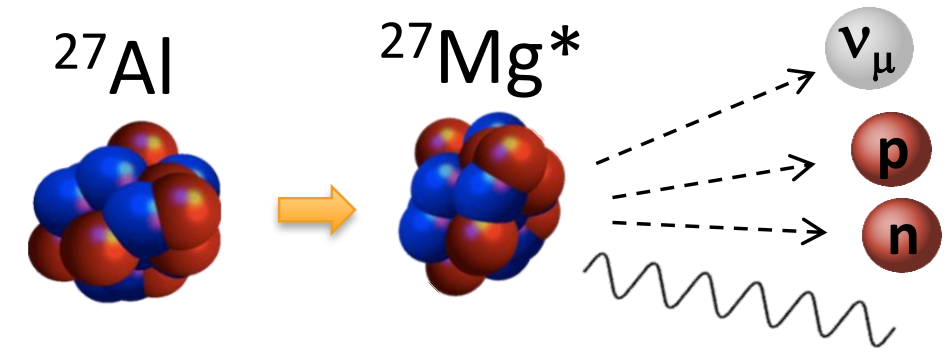




\section{Background(2): Prompt}

$>$ Radiative Pion Capture (RPC)

- $\pi^{-} N \rightarrow \gamma N^{\prime}, \gamma \rightarrow e^{+} e^{-}$ $\pi^{-} \mathrm{N} \rightarrow \mathrm{e}^{+} \mathrm{e}^{-} \mathrm{N}^{\prime}$

- $2 \%$ of captured $\pi^{-}$

- Mitigate by waiting

$>$ Beam and free decays

- Beam Electrons

- Muon Decay In Flight

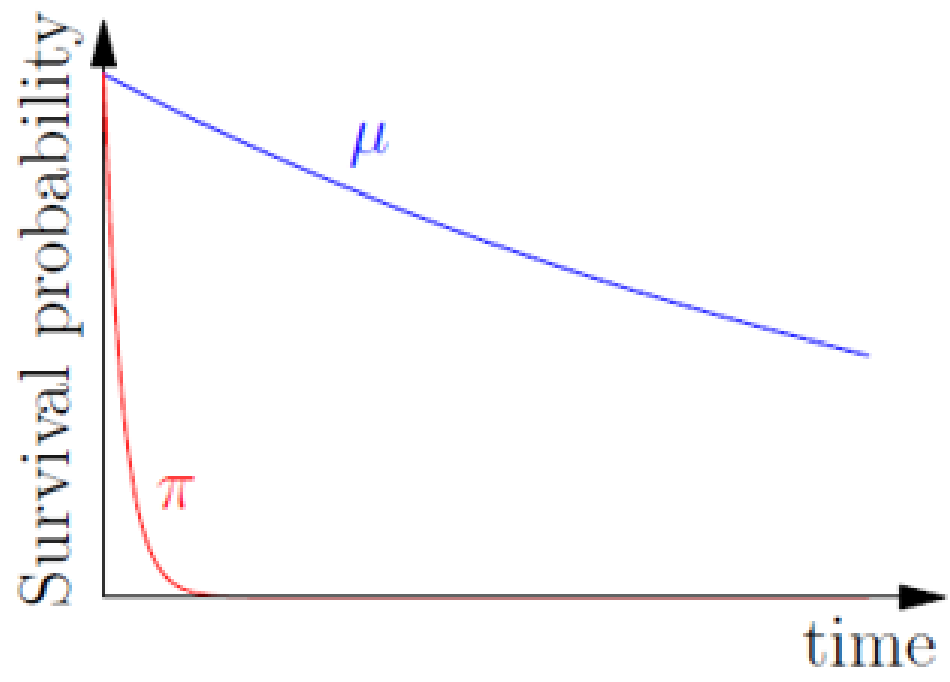

- Pion Decay In Flight

Delayed search time window help to mitigate prompt backgrounds 


\section{Background(3): Anti-protons}

$>$ The $8 \mathrm{GeV}$ proton is above anti-proton production threshold

- $\mathrm{pp} \rightarrow \overline{\mathrm{pp} p p}$,

- Anti-proton annihilate, produce $\pi^{0} \rightarrow \mathrm{e}^{+} \mathrm{e}^{-}$, $\pi^{-}$contribute to RPC backgrounds

- Anti-protons travel 10x more slowly

- Do not decay, time rejection does not help

Add absorbers at some locations in the muon beamline to absorb anti-protons

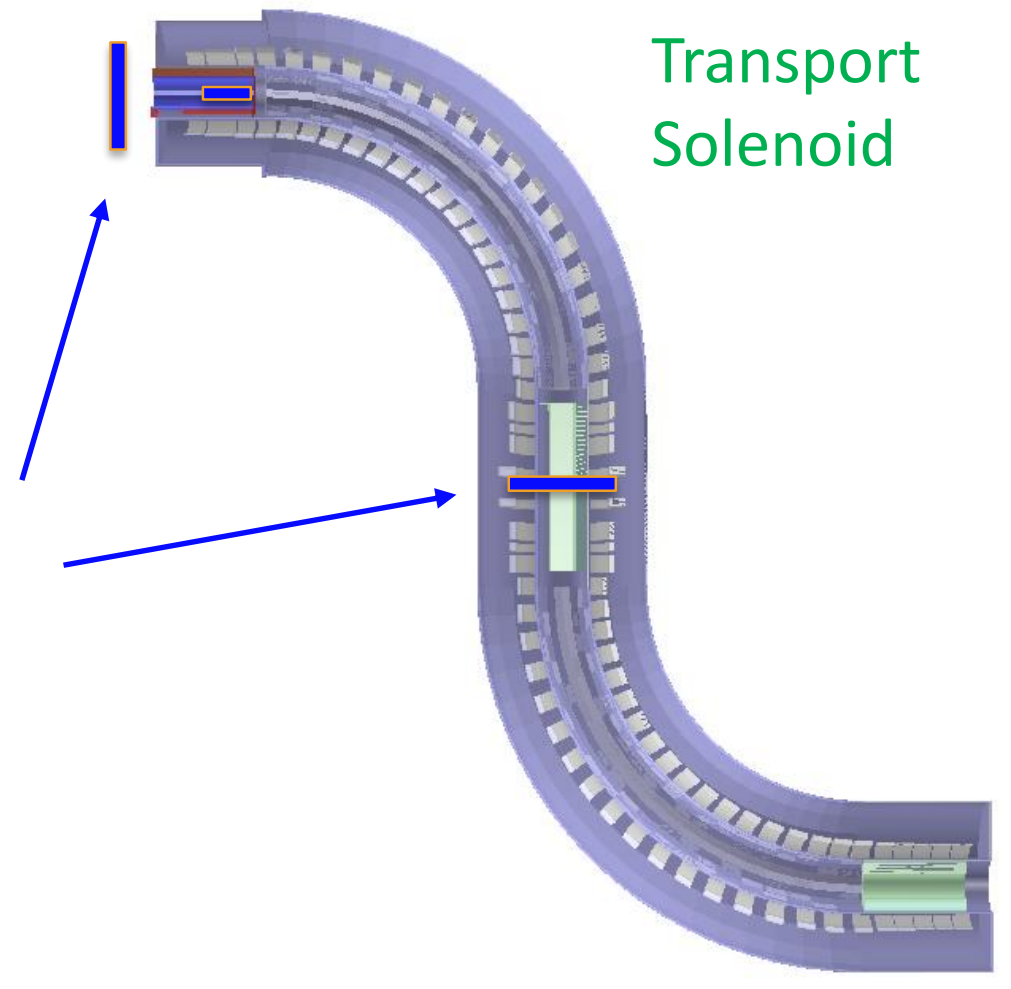




\section{Background(4): Cosmic Rays}

- Cosmic ray background $\sim 1$ event / day

- Requires $<10^{-4}$ inefficiency $\rightarrow 0.1$ event in 3 years

- Cosmic Ray Veto (CRV) made of 4 layers of overlapping scintillators

- Surrounding DS \& part of TS area

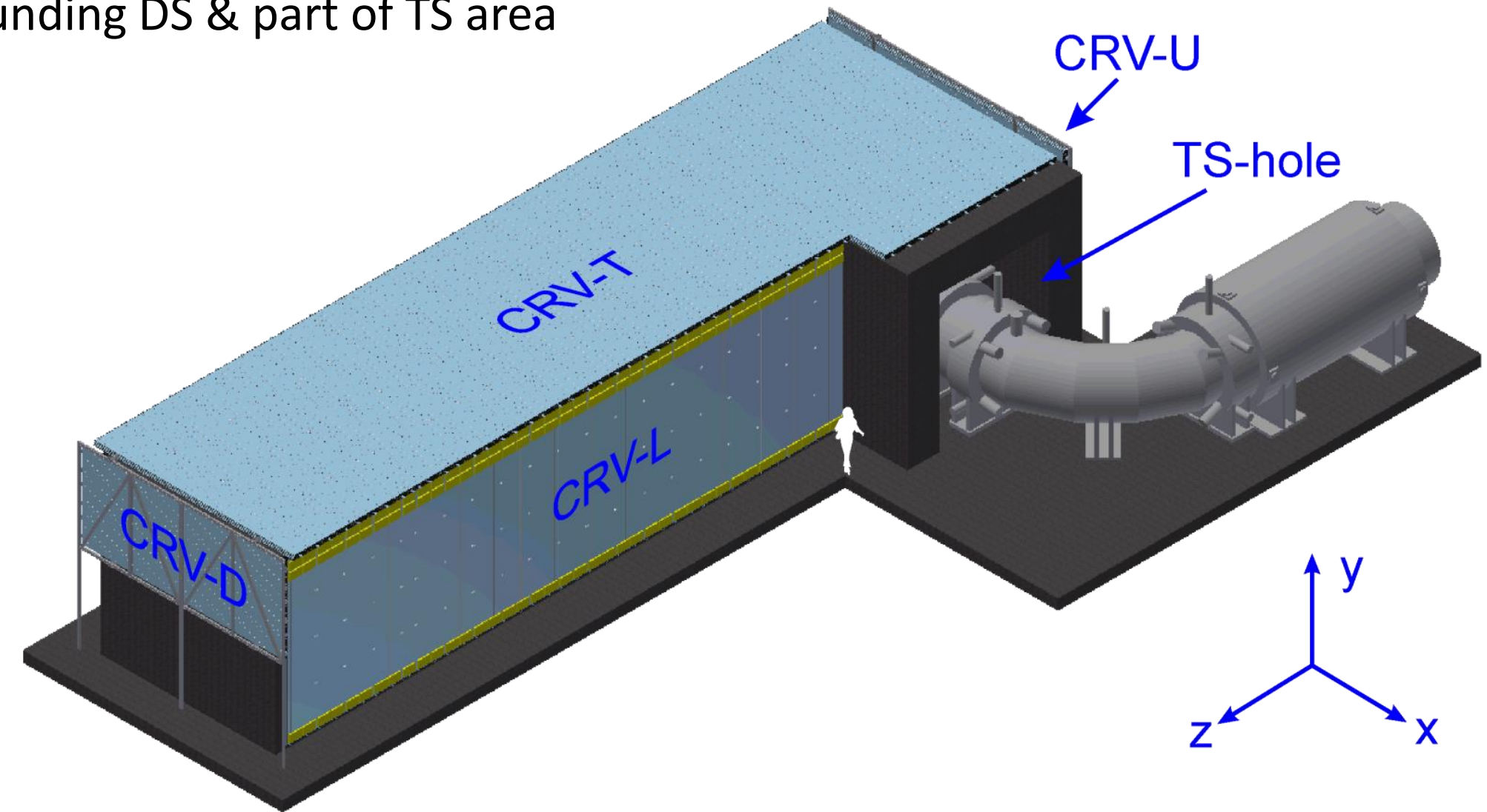




\section{All Backgrounds}

\begin{tabular}{|l|l|}
\hline Process & Expected event yield \\
\hline Cosmic rays & $0.209 \pm 0.022$ (stat) \pm 0.055 (syst) \\
DIO & $0.144 \pm 0.028$ (stat) \pm 0.11 (syst) \\
Antiprotons & $0.040 \pm 0.001$ (stat) \pm 0.020 (syst) \\
Pion capture & $0.021 \pm 0.001$ (stat) \pm 0.002 (syst) \\
Muon DIF & $<0.003$ \\
Pion DIF & $0.001 \pm<0.001$ \\
Beam electrons & $(2.1 \pm 1.0) \times 10^{-4}$ \\
RMC & $0.000_{-0.000}^{+0.004}$ \\
\hline Total & $0.41 \pm 0.13$ (stat + syst) \\
\hline
\end{tabular}

Numbers normalized to 3 years run, $3.6 \times 10^{20}$ POT events 


\section{Signal and Backgrounds}

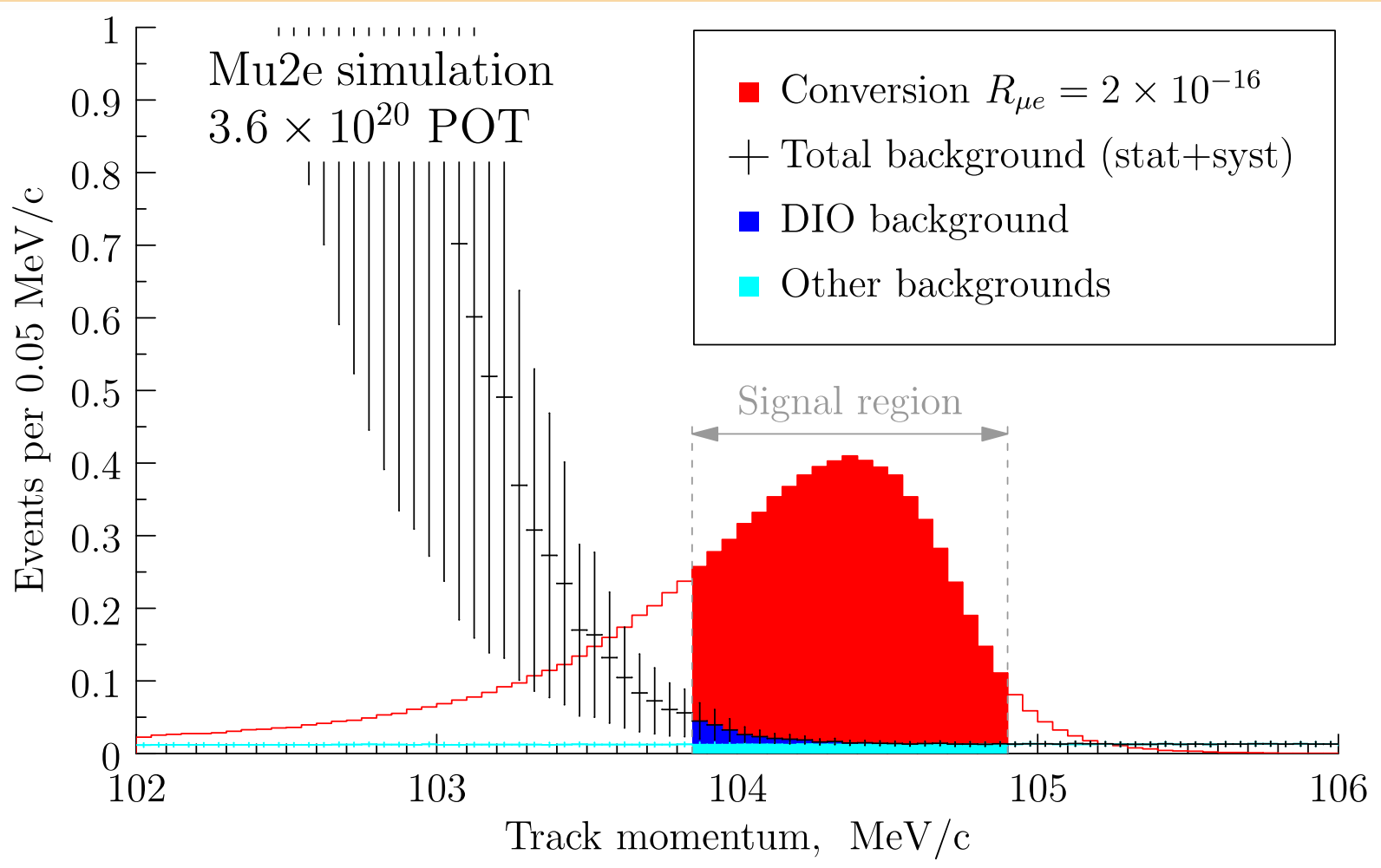

- Full Geant4 simulation and reconstruction, background overlaid with signal

- Discovery sensitivity accomplished with 3 years of running and suppressing backgrounds to $<0.4$ event total, $(5 \sigma) 2 \times 10^{-16}$

- $\quad$ Single event sensitivity $3 \times 10^{-17}$

- Null signal upper limit $8 \times 10^{-17} @ 90 \%$ C.L. 


\section{Mu2e Prototypes}
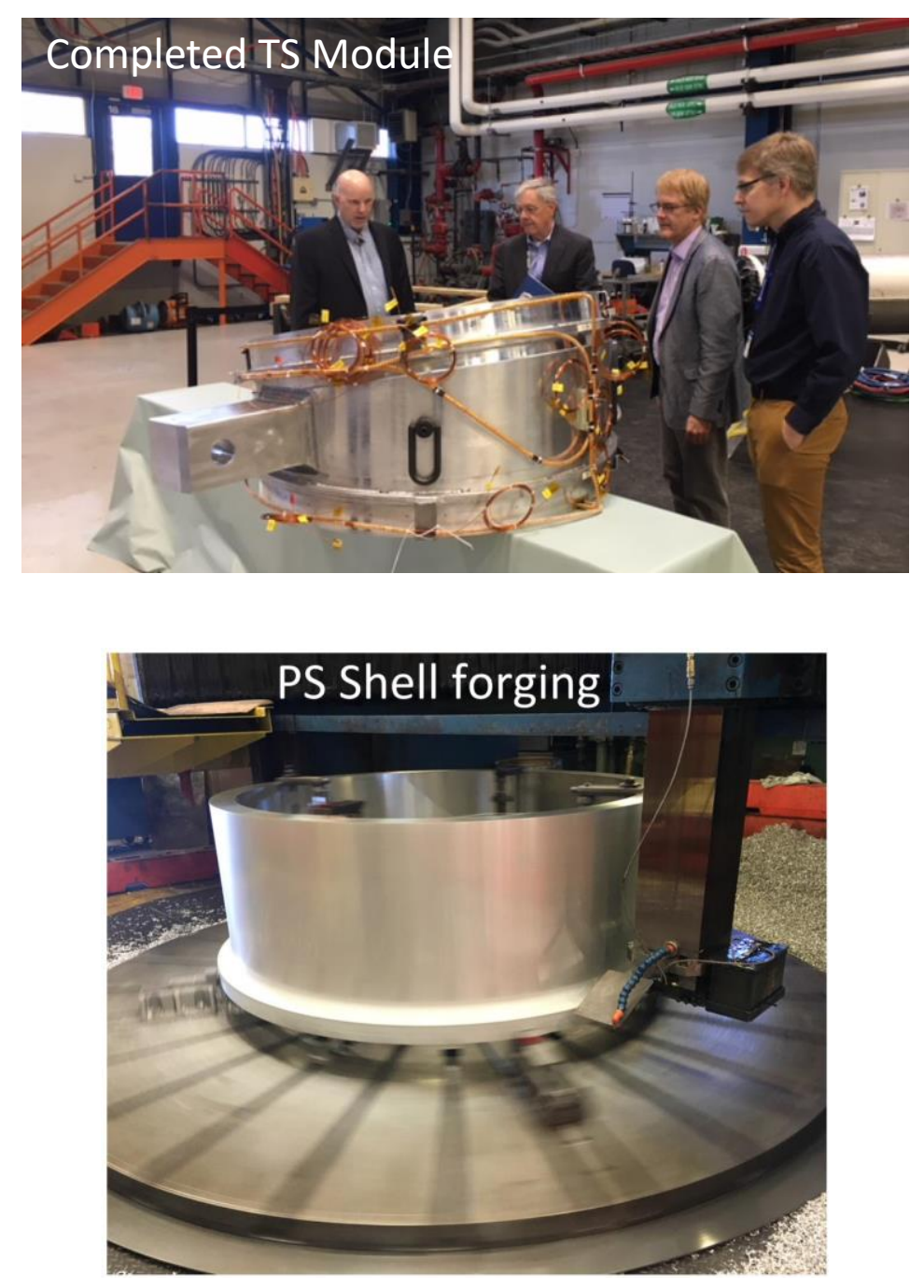
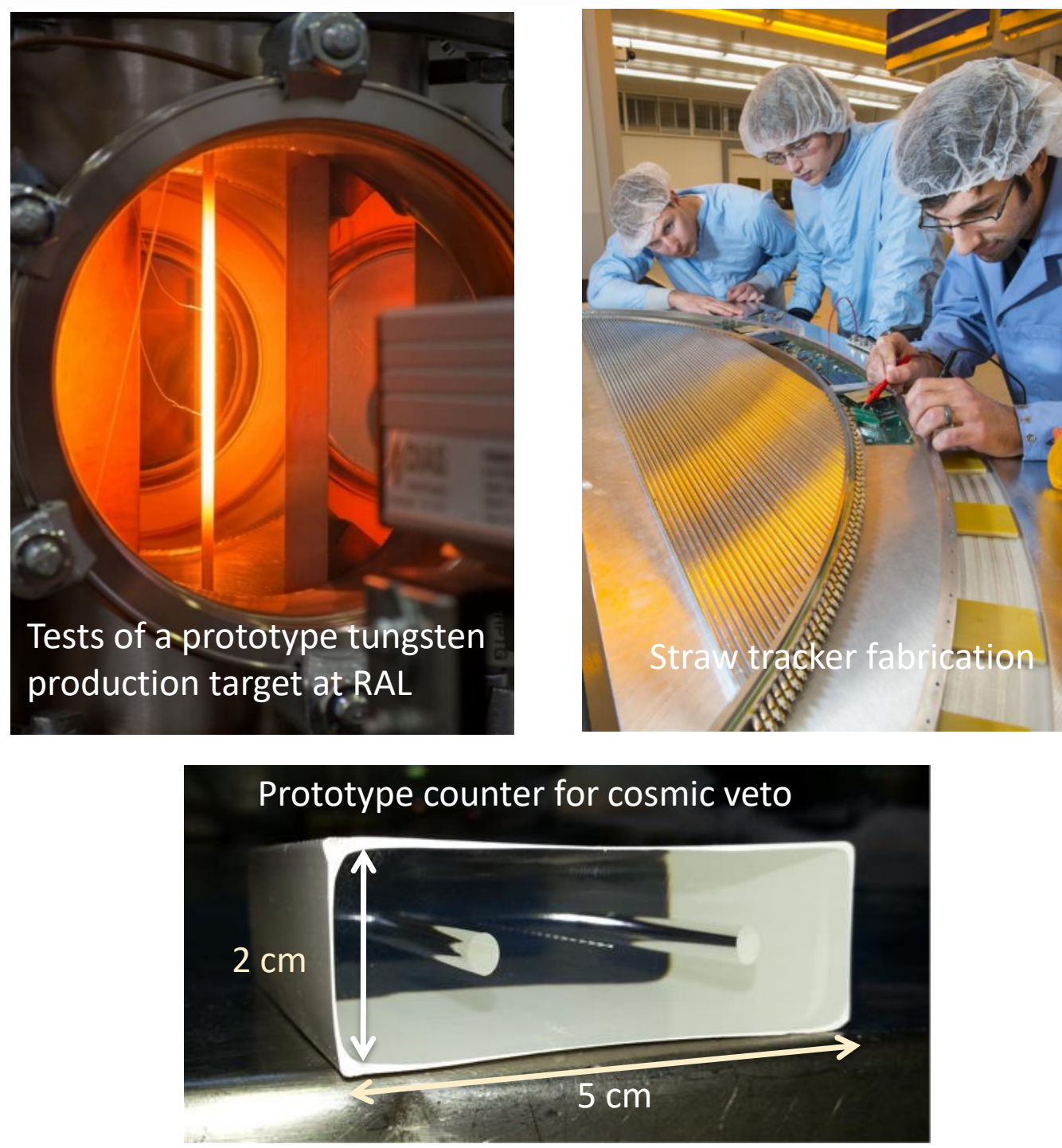

Zhengyun You (SYSU) 


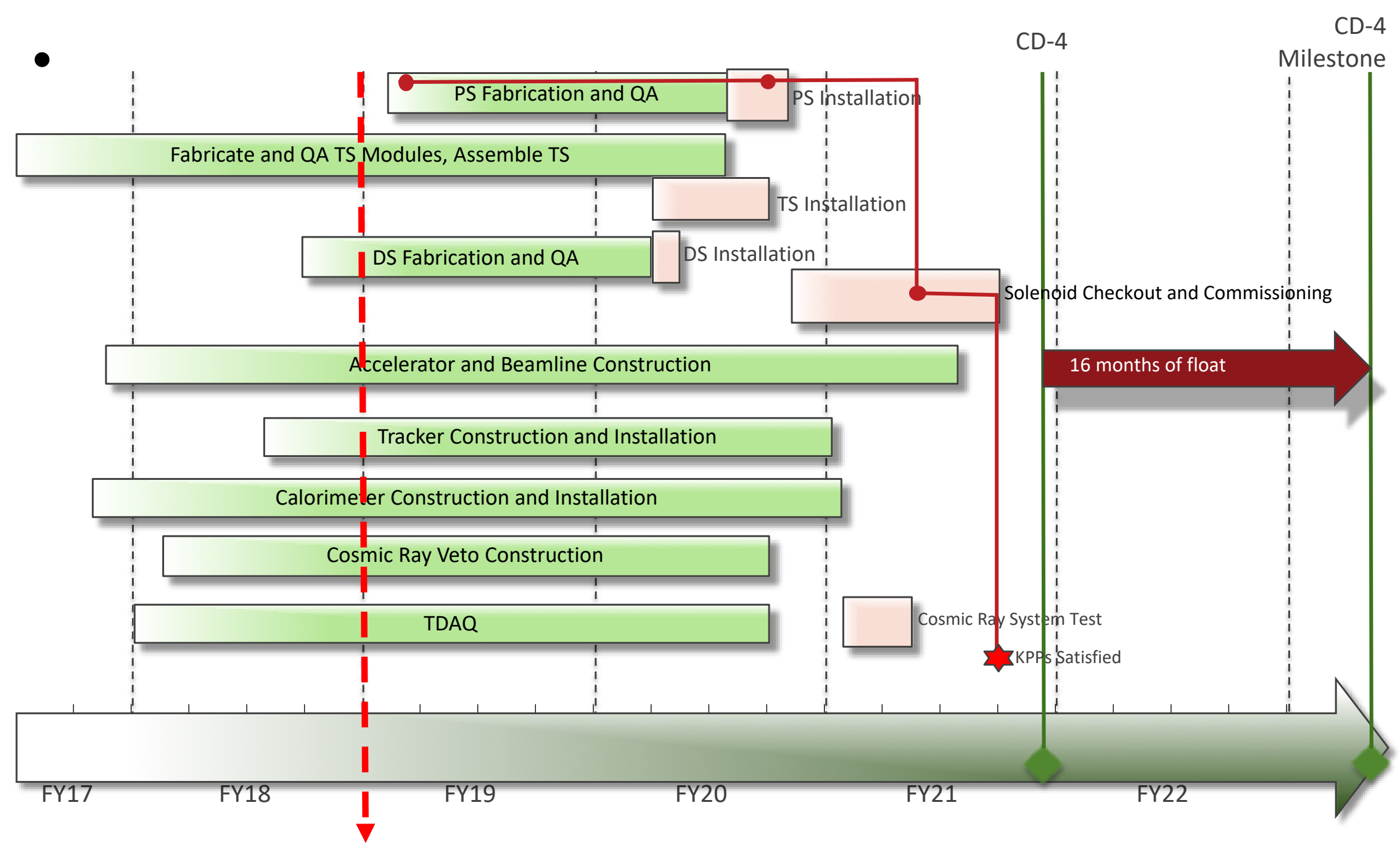

\section{Now}




\section{Mu2e II}

- If NO signal:

Higher sensitivity search;

X10 improvement

- Proton source upgrade

- Detector upgrade

- If signal:

Precision measurement;

Measure different target materials to discriminate between new physics models;

- Expression of interest (EOI) submitted to Fermilab PAC in 2018

(arXiv:1802.02599)

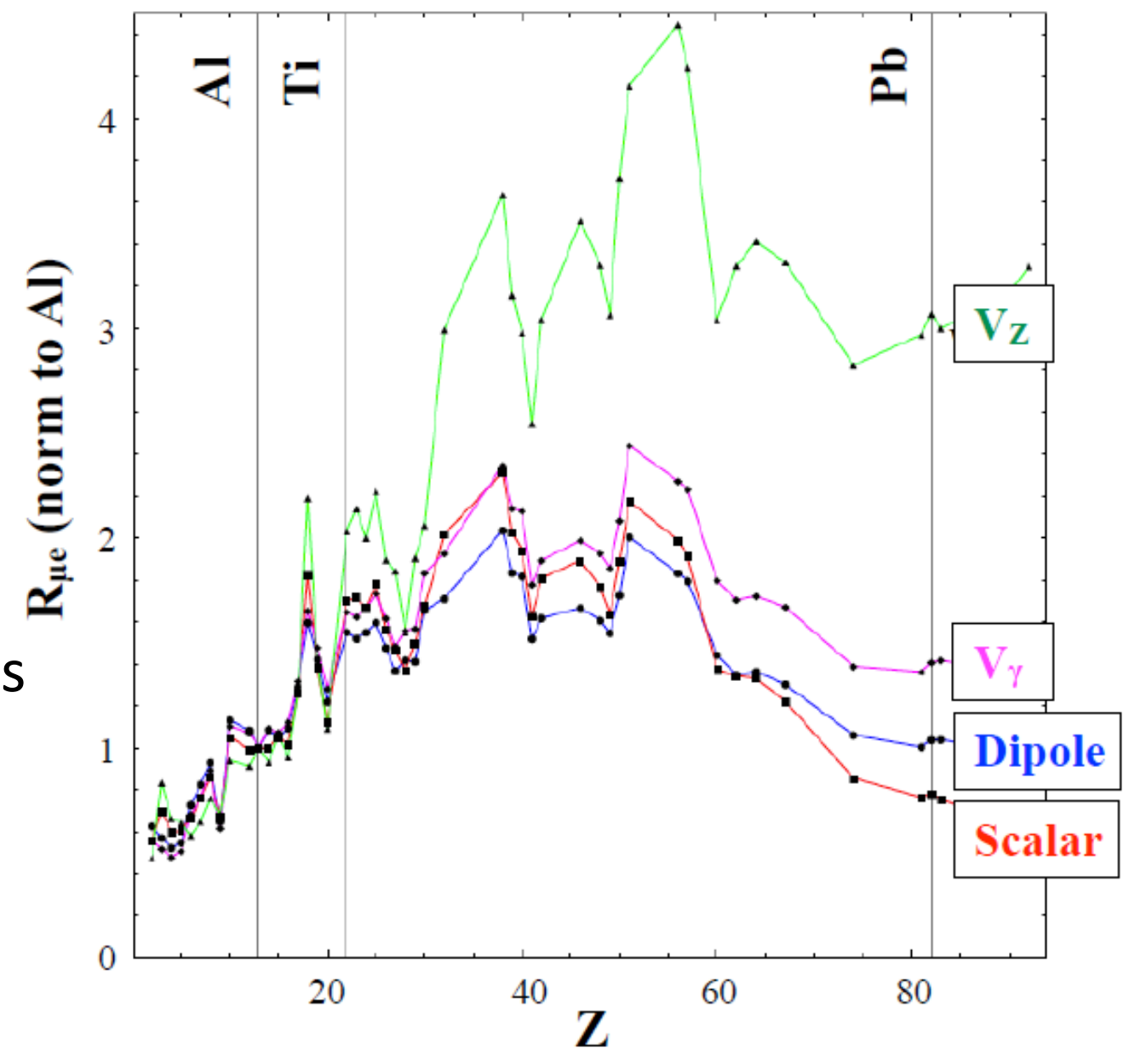

M. Koike et al., J. Phys. G29 (2003) 2051-2054 


\section{Summary}

$>$ CLFV has great potentials to probe new physics at the Intensity Frontier

$>$ Mu2e will search for CLFV with $\mu N \rightarrow e N$

- Address compelling science

- Single event sensitivity $R_{\mu e}=3 \times 10^{-17}$

- Improve the current limits by a factor of $10^{4}$

- Search for New Physics with mass scale up to $10^{4} \mathrm{TeV}$

$>$ Project is going well

- Complete construction in 2021

- Progressing on schedule for data taking 


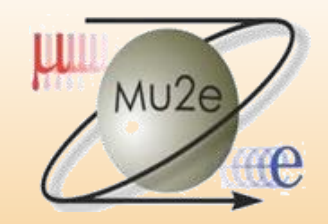

\section{Backup Slides}




\section{New Physics in $\mu \rightarrow e$}

\section{Supersymmetry}

:

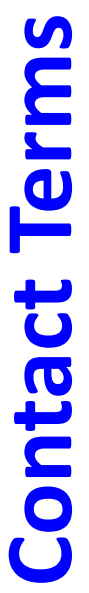

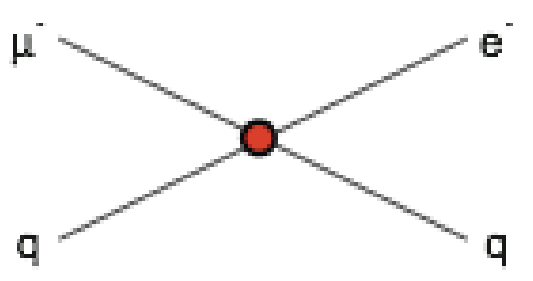
Heavy Neutrinos

$\left|\mathrm{U}_{\mu \mathrm{N}} \mathrm{U}_{\mathrm{eN}}\right|^{2} \sim 8 \times 10^{-13}$

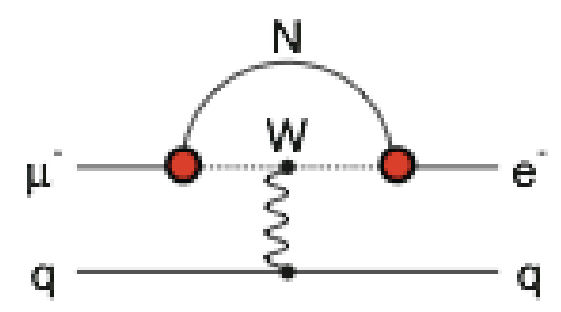

Leptoquark $\mathrm{M}_{\mathrm{LQ}}=$

$$
\Lambda_{\mathrm{c}} \sim 3000 \mathrm{TeV}
$$

\section{,}

Compositeness$$
3000\left(\lambda_{\mu \mathrm{d}} \lambda_{\text {ed }}\right)^{1 / 2} \mathrm{TeV} / \mathrm{c}^{2}
$$

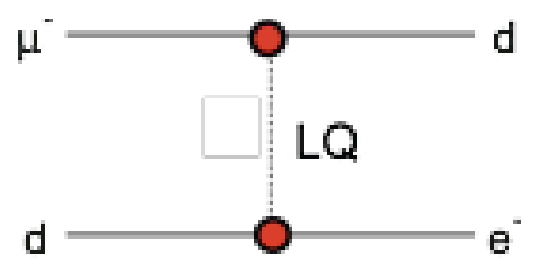

\section{Second Higgs Doublet}

$$
g\left(H_{\mu \mathrm{e}}\right) \sim 10^{-4} g\left(H_{\mu \mu}\right)
$$

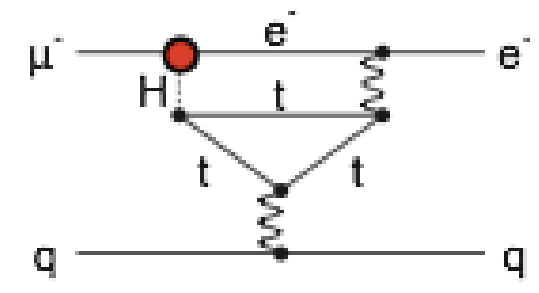

\section{Heavy Z'} Anomal. Z Coupling

$$
M_{z^{\prime}}=3000 \mathrm{TeV} / \mathrm{c}^{2}
$$

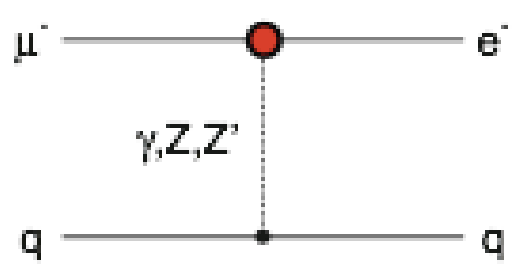

Flavor physics of leptons and dipole moments, arXiv:0801.1826 


\section{Mu2e Status}

- TDR published in Oct. 2014

- http://arXiv.org/abs/1501.05241

- DOE CD-2/3b received in March 2015

- DOE CD-3 awarded in July 2016

- First beam delivered in 2017 (Muon g-2)

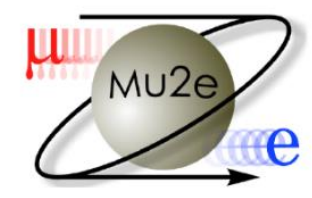

Mu2e Technical Design

Report

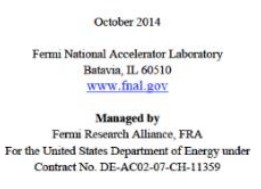

- Detector construction starts in 2018

OEENERGY

\section{Summary of Scenarios}

Mu2e keeps as priority funding on

\begin{tabular}{|c|c|c|c|c|c|c|c|c|c|}
\hline \multirow[b]{2}{*}{ Project/Actlvity } & \multicolumn{3}{|c|}{ Scenarlos } & \multicolumn{5}{|c|}{ Sclence Drlvers } & \multirow{2}{*}{ 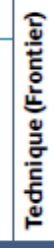 } \\
\hline & Scenarlo A & Scenarlo B & Senarlo C & 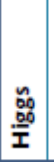 & 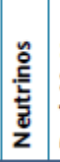 & 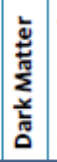 & & & \\
\hline \multicolumn{10}{|l|}{ Large Projects } \\
\hline Muon program: Mu2e, Muon g-2 & $Y$, Muze small reprofofie & $\mathrm{Y}$ & $Y$ & & & & & $\checkmark$ & $\mathrm{I}$ \\
\hline $\mathrm{HL}-\mathrm{LHC}$ & $\mathrm{Y}$ & Y & $\mathrm{Y}$ & $\checkmark$ & & $\checkmark$ & & $\checkmark$ & E \\
\hline LBNF + PIP-II & 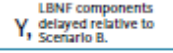 & $\mathrm{Y}$ & $\mathrm{Y}$, enhanced & & $\checkmark$ & & & $\checkmark$ & $\mathrm{I}, \mathrm{C}$ \\
\hline ILC & R\&D only & 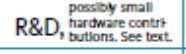 & $\mathrm{Y}$ & $\checkmark$ & & $\checkmark$ & & $\checkmark$ & $\mathrm{E}$ \\
\hline NuSTORM & $\mathrm{N}$ & $\mathrm{N}$ & $\mathrm{N}$ & & $\checkmark$ & & & & $\mathrm{I}$ \\
\hline RADAR & $\mathrm{N}$ & $\mathrm{N}$ & $\mathrm{N}$ & & $\checkmark$ & & & & 1 \\
\hline
\end{tabular}

Report of the Particle Physics Project Prioritization Panel May 2014 